\title{
Which Continuous-time Model Is Most Appropriate For Exchange Rates?
}

\author{
Deniz Erdemlioglu, \\ Sébastien Laurent \\ and \\ Christopher J. Neely
}

\author{
Working Paper 2013-024E \\ https://doi.org/10.20955/wp.2013.024
}

July 2015

\author{
FEDERAL RESERVE BANK OF ST. LOUIS \\ Research Division \\ P.O. Box 442 \\ St. Louis, MO 63166
}

The views expressed are those of the individual authors and do not necessarily reflect official positions of the Federal Reserve Bank of St. Louis, the Federal Reserve System, or the Board of Governors.

Federal Reserve Bank of St. Louis Working Papers are preliminary materials circulated to stimulate discussion and critical comment. References in publications to Federal Reserve Bank of St. Louis Working Papers (other than an acknowledgment that the writer has had access to unpublished material) should be cleared with the author or authors. 


\title{
Which continuous-time model is most appropriate for exchange rates? ${ }^{\text {th }}$
}

\author{
Deniz Erdemlioglu ${ }^{\mathrm{a}, *}$, Sébastien Laurent ${ }^{\mathrm{b}}$, Christopher J. Neely ${ }^{\mathrm{c}}$ \\ ${ }^{a}$ IÉSEG School of Management (LEM-CNRS), 3 Rue de la Digue, Lille, France. \\ ${ }^{b}$ Aix-Marseille Graduate School of Management-IAE, France. \\ ${ }^{c}$ Research Division, Federal Reserve Bank of St. Louis, USA.
}

\begin{abstract}
This paper evaluates the most appropriate ways to model diffusion and jump features of high-frequency exchange rates in the presence of intraday periodicity in volatility. We show that periodic volatility distorts the size and power of conventional tests of Brownian motion, jumps and (in)finite activity. We propose a correction for periodicity that restores the properties of the test statistics. Empirically, the most plausible model for 1-minute exchange rate data features Brownian motion and both finite activity and infinite activity jumps. Test rejection rates vary over time, however, indicating time variation in the data generating process. We discuss the implications of results for market microstructure and currency option pricing.
\end{abstract}

Keywords: Exchange rates, Brownian motion, Volatility, Jumps, Intraday periodicity, High-frequency data

JEL: C15, F31, G01

\section{Introduction}

What statistical model best describes the evolution of asset prices? Bachelier (1900) provided a very early attempt to answer this question when he modeled stock returns with "Brownian motion" (BM). More recently, the seminal work of Merton (1976) turned researchers' attention to modeling asset price jumps. The efficient markets hypothesis implies that asset returns exhibit limited predictability and that asset prices react rapidly to news surprises to prevent risk-adjusted profit opportunities. Thus, asset prices are likely to exhibit both continuous changes (diffusion) and discontinuous responses (jumps) to news. Decomposing returns into jumps and a diffusion with time-varying volatility is important because these two components imply different modeling and hedging strategies (Bollerslev and Todorov, 2011a,b). For example, although persistent time-varying diffusion volatility would help forecast future

\footnotetext{
The views expressed in this paper are solely those of the authors and do not necessarily reflect those of the Federal Reserve Bank of St. Louis or the Federal Reserve System.

${ }^{*}$ Corresponding author. IÉSEG School of Management, 3 Rue de la Digue, Lille, France. Tel: +33 (0)320 545892. Fax: +33(0)320 574855 .

Email addresses: d.erdemlioglu@ieseg.fr (Deniz Erdemlioglu), sebastien.laurent@univ-amu.fr (Sébastien Laurent), neely@stls.frb.org (Christopher J. Neely)
} 
volatility, jumps might contain no predictive information about volatility (Neely, 1999; Andersen et al., 2007a). Therefore, one must jointly model jumps and volatility to better explain asset price dynamics.

Our paper investigates which continuous-time model best describes intraday exchange rate fluctuations. We address the following questions: Does an appropriate model need BM or infinite activity jumps? Are jumps present in the exchange rates? If so, do exchange rate jumps have finite or infinite activity?

The first class of models that we consider is the Brownian SemiMartingale with Finite-Activity Jumps (BSMFAJ) class. These models incorporate a diffusion-Brownian-component to capture the continuous variation of the price process and a jump component to account for price discontinuities. ${ }^{1}$ These models exhibit finite-activity jump intensity - a finite number of jumps in any time interval. The compound Poisson process, which exhibits relatively rare and large jumps, is one example of this class. Andersen et al. (2007b) cite several authors who argue that the BSMFAJ class realistically models many asset prices.

BSMFAJ models may inappropriately restrict the jump dynamics of some asset prices, however. In particular, BSMFAJ models allow only relatively rare and large jumps, despite evidence of many small jumps in equities (Aït-Sahalia and Jacod, 2011; Lee and Hannig, 2010). Therefore, Madan et al. (1998) and Carr et al. (2002) have introduced a new class of models with infinite-activity Lévy processes for jumps: the Brownian SemiMartingale with Infinite-Activity Jumps (BSMIAJ) class (Cont and Tankov, 2004). ${ }^{2}$ The diffusion component of a BSMIAJ model captures the continuous price variation; and the jump component captures both rare and large jumps-potentially caused by important macro news (Dungey and Hvozdyk, 2012; Dungey et al., 2009; Lahaye et al., 2011) - as well as smaller, more frequent discontinuities that create risk for high-frequency trading strategies (Aï-Sahalia and Jacod, 2012). Jumps can arrive infinitely often in BSMIAJ models, which might better describe the data than the combination of BM and Poisson jumps (see Geman, 2002; Li et al., 2012, 2008). ${ }^{3}$

The choice of the most appropriate model has important implications for empirical asset pricing and equity research. For instance, a Brownian SemiMartingale (BSM) model-with only continuous components - generates different risk measures (and premia) than BSMFAJ or BSMIAJ models (Bollerslev and Todorov, 2011a,b; Drechsler and Yaron, 2011). Consumption-based asset pricing mod-

\footnotetext{
${ }^{1}$ Semimartingale models are quite useful in financial economics and continuous-time finance because they rule out arbitrage opportunities (see Back, 1991).

${ }^{2}$ The literature studying infinite-activity Lévy processes is young but growing rapidly. Examples include the works of Aït-Sahalia and Jacod (2009a,b, 2010, 2012), Todorov and Tauchen (2006), Aït-Sahalia (2004), Carr and Wu (2003, 2004, 2007), Lee and Hannig (2010), Huang and Wu (2004), Carr and Madan (1998), and Bakshi et al. (2008), among others.

${ }^{3}$ It is worth noting that BSMIAJ models can exclude the finite-activity jump component so that only the infiniteactivity jump component represents the price discontinuities.
} 
els (C-CAPM) rely on the continuous part of the returns and hence characterize only the diffusion risk (see Back, 1991). ${ }^{4}$ As Maheu et al. (2013) show, jumps significantly contribute to the equity risk premium.

Few papers have investigated the best continuous-time model for exchange rates. Todorov and Tauchen (2010) and Cont and Mancini (2011) are most closely related to our paper; these works have also studied the characteristics of foreign-exchange-data-generating processes, focusing on jump activity and jump variation, respectively. Specifically, both papers use the Blumenthal-Getoor index to estimate jump activity and find that BM is present in foreign exchange data. We confirm this with power variation measures. We also confirm Cont and Mancini (2011)'s finding of finite jump intensity. ${ }^{5}$

Our work extends and complements these studies. Methodologically, we explicitly account for the effect of intraday periodicity in volatility on the flexible testing methodology of Aït-Sahalia and Jacod (2012). Our simulations show that intraday periodicity in volatility can badly distort the size and power of ASJ tests for Brownian motion, jumps, no-jumps, finite and infinite activity jumps. We propose a correction that removes the intraday periodicity in volatility and restores the desired properties of the tests.

We then apply these corrected tests to characterize the properties of a 10-year sample of 1-minute data on three major exchange rates. Unlike the (jump) index estimation approaches of Todorov and Tauchen (2010) and Cont and Mancini (2011), we test the presence of each potential component separately (i.e., BM, jumps, and jump activity). These modified tests and long span of 1-minute data yield new insights into the foreign-exchange-data-generating process. For example, Todorov and Tauchen (2010) conclude that the Brownian-plus-Poisson-jumps model might be misspecified, although they stress that this inference depends on the sampling frequency and testing technique. We show that a combination of Brownian motion, finite activity jumps and infinite activity jumps better characterize 1-minute foreign exchange data than simpler, two-component models. Importantly, we also show that the test rejection rates display significant time variation, indicating that the most appropriate model of the data has probably changed over time, with more rejections of jumps and finite activity jumps. The long span of data was crucial to examine the previously neglected subject of time variation in the data generating process.

We organize our paper as follows: Section 2 presents the methodology. Section 3 describes the

\footnotetext{
${ }^{4}$ See also Lustig and Verdelhan (2007), who present empirical evidence on the link between aggregate consumption growth and exchange rate dynamics.

${ }^{5}$ See Bates (1996b), who propose a parametric model that captures stochastic volatility and jumps of deutsche mark options.
} 
exchange rate data and extends the base technique to account for intraday periodicity. In Section 4 , we conduct simulations to assess the finite sample properties of our testing procedures. Section 5 reports the empirical results and discusses the implications. Section 6 concludes.

\section{The base methodology}

\subsection{Theoretical background}

In line with previous literature, we assume that the log-price $X(t)$ follows a Brownian semimartingale with jumps such that

$$
d X(t)=\underbrace{\mu(t) d t}_{\text {drift }}+\underbrace{\sigma(t) d W(t)}_{\text {continuous component }}+\operatorname{JUMPS}(t), t \geq 0
$$

where $d X(t)$ denotes the logarithmic price increment, $\mu(t)$ is a continuous, locally bounded, variation process, $\sigma(t)$ is a strictly positive and càdlàg (right-continuous with left limits) stochastic volatility process, and $W(t)$ is a standard BM. The JUMPS component of (1) potentially represents both finiteand infinite-activity jumps. That is,

$$
\operatorname{JUMPS}(\mathrm{t}):=\underbrace{\kappa(t) d q(t)}_{\text {finite activity }}+\underbrace{h(t) d L(t)}_{\text {infinite activity }}
$$

where $q(t)$ denotes a counting process (e.g., Poisson process), $L(t)$ is a pure Lévy jump process (e.g., a Cauchy process), and $\kappa(t)$ and $h(t)$ denote the jump sizes of the counting and Lévy processes, respectively.

In the presence of noise, the true value of the log price is $X(t)$ but in the data we observe $Z(t)$. That is,

$$
Z(t)=X(t)+e(t)
$$

where $e(t)$ is the additive noise term as in Aït-Sahalia et al. (2012).

We assume that the log-price process (either $X(t)$ or $Z(t)$ ) is observed at discrete points in time. The continuously compounded $i$ th intraday return of a trading day $t$ is therefore given by $r_{t, i} \equiv$ $X(t+i \Delta)-X(t+(i-1) \Delta)$ or $r_{t, i} \equiv Z(t+i \Delta)-Z(t+(i-1) \Delta)$, with $i=1, \ldots, M$ and trading days $t=1, \ldots, T$. Let $M \equiv\lfloor 1 / \Delta\rfloor$ denote the number of intraday observations over the day; $\Delta=1 / M$ is the time between consecutive observations, the inverse of the observation frequency. In the absence noise and of the JUMPS component, (1) is a BSM model and therefore the realized variance, i.e. 
$R V_{t}=\sum_{i=1}^{M} r_{t, i}^{2}$, is a consistent estimator (when $\Delta \rightarrow 0$ ) of the integrated variance

$$
I V_{t} \equiv \int_{t-1}^{t} \sigma^{2}(s) d s
$$

Some of the test statistics used in this paper are functions of "truncated power variations", a class of statistics that depend on three parameters: $p$, the power exponent, $u$, the truncation parameter, and $k$, the sampling frequency parameter. Ait-Sahalia and Jacod (2010) show that one can infer the properties of the data generating process from the probability limits of truncated power variations under combinations of these three parameters. We now define the power variation statistics introduced in Ait-Sahalia and Jacod (2010).

Definition. Denote the truncated power variation as $\widehat{B}(p, u, \Delta)_{t}$. That is,

$$
\widehat{B}(p, u, \Delta)_{t}:=\sum_{i=1}^{1 / \Delta}\left|r_{t, i}\right|^{p} 1_{\left\{\left|r_{t, i}\right| \leq u\right\}},
$$

where $u=\alpha \Delta^{\varpi}$ is the truncation threshold and $\alpha(>0)$ is expressed in units of standard deviations of the continuous part of the process for a constant $\varpi \in(0,1 / 2)$. The truncation function on the right-hand side of (4) can be used to exclude large changes (i.e., jumps) from the calculation, which allows us to calculate moments of the diffusion process while excluding jumps. Note that $\widehat{B}(2, \infty, \Delta)_{t}$ corresponds to the realized variance estimator presented above.

In summary, these truncated power variation functions compute statistics of diffusion returns and "large" returns (i.e., jumps) that allow us to test the hypotheses described in the next section.

\subsection{Hypotheses and test statistics}

This subsection follows Ait-Sahalia and Jacod (2012) in presenting the statistics to test (on a daily basis) five hypotheses about the characteristics of high-frequency exchange rates:
(A) $H_{0}$ : Brownian motion is present
vs. $H_{1}$ : Brownian motion is not present
(B) $H_{0}$ : Jumps are not present
vs. $H_{1}$ : Jumps are present
(C) $H_{0}$ : Jumps are present
vs. $H_{1}$ : Jumps are not present
(D) $H_{0}$ : Jumps have finite activity
vs. $H_{1}$ : Jumps have infinite activity
(E) $H_{0}$ : Jumps have infinite activity
vs. $H_{1}$ : Jumps have finite activity

(A) - To test for BM:

$$
\widehat{S}_{W}(p, u, k, \Delta)_{t}=\frac{\widehat{B}(p, u, \Delta)_{t}}{\widehat{B}(p, u, k \Delta)_{t}} \stackrel{\mathbb{P}}{\longrightarrow}\left\{\begin{array}{cl}
k^{1-p / 2}, & X \text { has Brownian motion on }[t-1, t] \\
1, & X \text { has no Brownian motion on }[t-1, t] \\
k, & \text { noise dominates on }[t-1, t],
\end{array}\right.
$$


where $k$ is the sampling scale parameter. Under $H_{0}, \frac{\widehat{S}_{W}(p, u, k, \Delta)_{t}-k^{1-p / 2}}{{\sqrt{\widehat{V}_{t}^{w}}}^{w}} \sim N(0,1)$, where $\widehat{V}_{t}^{w}$, an estimate of the asymptotic variance of $\widehat{S}_{W}(p, u, k, \Delta)_{t}$, is given in (B.2).

(B)-(C) - To test for no jumps and for jumps:

$$
\widehat{S}_{J}(p, k, \Delta)_{t}=\frac{\widehat{B}(p, \infty, k \Delta)_{t}}{\widehat{B}(p, \infty, \Delta)_{t}} \stackrel{\mathbb{P}}{\longrightarrow}\left\{\begin{array}{cl}
1, & X \text { has jumps on }[t-1, t] \\
k^{p / 2-1}, & X \text { is continuous on }[t-1, t] \\
1 / k, & \text { noise dominates on }[t-1, t]
\end{array}\right.
$$

Under $H_{0}$ : no jumps, $\frac{\widehat{S}_{J}(p, k, \Delta)_{t}-k^{p / 2-1}}{\sqrt{\widehat{V}_{t}^{c}}} \sim N(0,1)$, where $\widehat{V}_{t}^{c}$, an estimate of the asymptotic variance of $\widehat{S}_{J}(p, u, k, \Delta)_{t}$ under the null of no jumps, is given in (B.5). Under $H_{0}$ : jumps, $\frac{\widehat{S}_{J}(p, k, \Delta)_{t}-1}{\sqrt{\widehat{V}_{t}^{j}}} \sim N(0,1)$, where $\widehat{V}_{t}^{j}$, an estimate of the asymptotic variance of $\widehat{S}_{J}(p, u, k, \Delta)_{t}$ under the null of jumps, is given in (B.9).

(D) - To test for finite-activity jumps and/or BM vs. infinite activity jumps:

$$
\widehat{S}_{F A}(p, u, k, \Delta)_{t}=\frac{\widehat{B}(p, u, k \Delta)_{t}}{\widehat{B}(p, u, \Delta)_{t}} \stackrel{\mathbb{P}}{\longrightarrow}\left\{\begin{array}{cl}
k^{p / 2-1}, & X \text { has finitely many jumps on }[t-1, t] \\
1, & X \text { has infinitely many jumps on }[t-1, t] \\
1 / k, & \text { noise dominates on }[t-1, t] .
\end{array}\right.
$$

Under $H_{0}$ : finite activity (FA) jumps, $\frac{\widehat{S}_{F A}(p, u, k, \Delta)_{t}-k^{p / 2-1}}{\sqrt{\widehat{V}_{t}^{F A}}} \sim N(0,1)$, where $\widehat{V}_{t}^{F A}$, an estimate of the asymptotic variance of $\widehat{S}_{F A}(p, u, k, \Delta)_{t}$ under the null of FA jumps (or BM), is given in (B.12). Note that the null hypothesis embeds cases where the process has only BM component and the case with BM and FA jumps.

(E) - To test for infinite-activity jumps vs. finite-activity jumps and/or BM:

$\widehat{S}_{I A}\left(p, p^{\prime}, u, \gamma, \Delta\right)_{t}=\frac{\widehat{B}\left(p^{\prime}, \gamma u, \Delta\right)_{t} \widehat{B}(p, u, \Delta)_{t}}{\widehat{B}\left(p^{\prime}, u, \Delta\right)_{t} \widehat{B}(p, \gamma u, \Delta)_{t}} \stackrel{\mathbb{P}}{\longrightarrow}\left\{\begin{array}{cl}\gamma^{p^{\prime}-p}, & X \text { has infinitely many jumps on }[t-1, t] \\ 1, & X \text { has finitely many jumps on }[t-1, t]\end{array}\right.$

where $\gamma>1$ and $p^{\prime}$ is another parameter for power variation such that $p^{\prime}>p>2$. Note that while (5) requires $p<2$, the test statistic in (6) identifies the presence of jumps for $p>2$. Under $H_{0}$ : infinite activity (IA) jumps, $\frac{\widehat{S}_{I A}\left(p, p^{\prime}, u, \gamma, \Delta\right)_{t}-\gamma^{p^{\prime}-p}}{\sqrt{\widehat{V}_{t}^{I A}}} \sim N(0,1)$, where $\widehat{V}_{t}^{I A}$, an estimate of the asymptotic variance of $\widehat{S}_{I A}\left(p, p^{\prime}, u, \gamma, \Delta\right)_{t}$ under the null of IA jumps, is given in (B.16). Note that the alternative hypothesis embeds both the case with only BM and the case with both FA jumps and BM. All tests but test (C) are one-sided left-tailed tests. 


\section{An extension: the role of intraday periodicity}

The previous section presents the tools used to identify the most appropriate continuous-time model for exchange rates. This section extends this methodology to account for intraday periodicity in volatility. ${ }^{6}$ To do so, we first illustrate the presence of periodicity in the real intraday data, then we use simulations to establish that intraday periodicity in volatility may distort the properties of test statistics. Finally, we propose a simple correction to restore the properties of these tests.

\subsection{Data}

We use 1-minute data for the EUR/USD, USD/JPY, and GBP/USD exchange rates from January 1, 2000, to March 12, 2010. Disk Trading provides the last mid-quotes (average of the logarithms of bid and ask quotes) of 1-minute intervals throughout the 24-hour trading day. Following Andersen and Bollerslev (1998a), one trading day extends from 16:01 EST on day $t-1$ to 16:00 EST on day $t$.

As is usual in the literature, we omit trading days with too many missing values or low trading activity. Similarly, we deleted weekends plus certain fixed and irregular holidays, trading days for which there are more than 360 missing values at the 1-minute frequency (corresponding to more than one fourth of the data), and trading days with too many empty intervals and consecutive prices. ${ }^{7}$ These criteria leave 2483, 2479 and 2472 days, respectively, for the EUR/USD, USD/JPY and the GBP/USD exchange rates.

\subsection{Intraday periodicity in volatility}

As Baillie and Bollerslev (1991) and Andersen and Bollerslev (1997) document, foreign exchange volatility shows strong intraday periodic effects caused by regular trading patterns, such as openings and closings of the three major markets: Asia, Europe and North America. Figure 1 illustrates the periodicity by showing mean absolute EUR/USD, USD/JPY and GBP/USD returns over the (1440) 1-minute intervals.

[ Insert Figure 1 about here ]

The figure indicates that volatility is low during the Far East market hours, from 16:00 EST (21:00 GMT) to 24:00 EST (05:00 GMT), but activity picks up as Europe begins to trade around 2:00 EST

\footnotetext{
${ }^{6}$ Previous studies have shown that periodicity matters (i) for estimating and forecasting intraday volatility (e.g., Andersen and Bollerslev, 1998b), (ii) for studying the impact of news on volatility (e.g., Dominquez and Panthaki, 2006; Hautsch et al., 2011), (iii) for estimating co-volatility (Boudt et al., 2011a), and (iv) for detecting intraday jumps (Boudt et al., 2011b).

${ }^{7}$ These holidays include the New Year (December 31 - January 2), Martin Luther King Day, Washington's birthday or Presidents' Day, Good Friday, Easter Monday, Memorial Day, Independence Day, Labor Day, Thanksgiving Day, and Christmas (December $24-26$ ).
} 
(7:00 GMT), and Far East activity begins to wane. The most active period is the overlap of the European and North American markets, between 7:00 EST and 11:00 EST. Volatility then declines, as first the European and then U.S. markets wind down. Asian markets reopen at around 16:00 EST $(21: 00 \mathrm{GMT}) .^{8}$

\section{Monte Carlo study}

This section describes our Monte Carlo study that shows how intraday patterns in volatility distort the properties of realized power variation and ASJ tests. We then propose a simple correction that restores the appropriate properties to the statistics.

\subsection{Simulation setup and data generating processes}

We apply the tests at a daily level and the total number or replications is 25,000 . We consider the following three data generating processes, each of which feature GARCH and intraday periodic patterns in volatility but different processes for jumps:

\section{- DGP1: GARCH diffusion process}

$$
\begin{aligned}
d X(t) & =\sigma(t) d W_{p}(t), \\
\sigma(t) & =f(\tau(t)) \sigma_{\text {garch }}(t), \\
d \sigma_{\text {garch }}^{2}(t) & =\theta\left[\omega-\sigma_{\text {garch }}(t)^{2}\right] d t+(2 \lambda \theta)^{1 / 2} \sigma_{\text {garch }}(t)^{2} d W_{d}(t), \\
\log f(\tau(t)) & =\sum_{j=1}^{4}\left(\gamma_{j} \cos (2 \tau(t) j \pi)+\delta_{j} \sin (2 \tau(t) j \pi)\right),
\end{aligned}
$$

where $f(\tau(t))$ is the periodicity factor; the $\cos$ and $\sin$ terms depend on the time of the day. Figure 2 plots the intraday periodicity patterns used in the simulations. ${ }^{9}$ For $d \sigma_{\text {garch }}^{2}(t)$, we follow Andersen and Bollerslev (1998b) and set $\theta=0.035, \omega=0.636$ and $\lambda=0.296$. The initial values are $X(0)=1$ and $\sigma_{\text {garch }}^{2}(0)=0.01$.

\section{[ Insert Figure 2]}

\section{- DGP2: GARCH diffusion process with finite-activity (FA) jumps}

$$
\begin{aligned}
d X(t) & =\sigma(t) d W_{p}(t)+\kappa(t) d q(t), \\
\kappa(t) & \sim \sigma(t) \sqrt{m}([-2,-1] \cup[1,2]), \\
d q(t) & \sim \operatorname{Poisson}(l),
\end{aligned}
$$

\footnotetext{
${ }^{8}$ This intraday pattern is consistent with those reported in the literature. See, e.g., Andersen and Bollerslev (1998b).

${ }^{9} \mathrm{We}$ use the estimated parameters $\gamma_{1}, \delta_{1}, \ldots, \gamma_{4}, \delta_{4}=(-0.24422,-0.49756,-0.054171,0.073907,-0.26098,0.32408$, -0.11591, -0.21442). $f(\tau(t))$ is rescaled such that its squares sum to one over one day.
} 
where $\sigma(t)$ is defined as in DGP1 while the (finite activity) jump size $\kappa(t)$ is modeled as the product between $\sigma(t)$ and a uniformly distributed random variable on $\sqrt{m}([-2,-1] \cup[1,2])$. The parameter $m$ determines the magnitude of the jumps. We set $m=0.5$ and choose $l$ such that there is one jump per day. We also conditioned the occurrence of jumps such that simulated jumps only occur when $f(\tau(t))<0.5$.

\section{- DGP3: GARCH diffusion process with infinite-activity (IA) jumps}

$$
\begin{aligned}
d X(t) & =\sigma(t) d W_{p}(t)+f(\tau(t)) d L(t), \\
d L(t) & \sim C G M Y(C, G, M, Y),
\end{aligned}
$$

where $\sigma(t)$ is defined as in DGP1 while $L(t)$ is an infinite-activity jump process, that is, the CGMY model of Carr et al. (2002). In this process, $C$ measures the level of activity and controls the kurtosis, $G$ and $M$ are the decay parameters ( $G=M$ implies symmetry) and $Y$ determines the jump type (i.e. finite or infinite). To simulate data with this process, we set $C=5, G=3, M=3, Y=0.5$. $Y=0.5$ ensures that the jump activity is infinite, completely monotone with finite variation (which is the case for $0<Y<1$ ). We rescale the Levy component such that the quadratic variation of the Brownian motion (BM) part (i.e. $\sigma(t) d W_{p}(t)$ ) over the quadratic variation of the Levy component (i.e. $f(\tau(t)) d L(t))$ is $15 \%$ every day.

\subsection{The impact of periodicity on the realized power variation}

Section 2.2 presents statistics - Equations (5) to (8) - that are functions of truncated power variations. The level of truncation is chosen as a multiple of diffusion standard deviation to differentiate jumps from diffusion returns. If diffusion volatility varies over the day, however, than the statistics in Section 2.2 will not consistently differentiate jumps from diffusion returns. During periods of low (high) diffusion volatility, a constant level of truncation will be relatively high (low) and statistics in (5) to (8) will have poor properties. ${ }^{10}$

We investigate the effect of periodicity in volatility on the truncation mechanism of the power variation statistics. To illustrate the potential problem, we simulate data using DGP1 and report the frequency of truncated observations per intraday interval. Aït-Sahalia and Jacod (2012) express $u$ as $\alpha \Delta^{\varpi}$ (i.e., Equation (4) for deriving the asymptotic properties of their tests but in their simulations and empirical applications, they express it as $u=a_{0} \sqrt{I V_{t}} \Delta^{0.5}$, i.e. $a_{0}$ times the spot volatility under the assumption of constant volatility through the day. Monte Carlo simulations of Aït-Sahalia and Jacod

\footnotetext{
${ }^{10}$ Note that truncation occurs when $\left|r_{t, i}\right|>u$.
} 
(2012) suggest a set of plausible values for $a_{0}$ ranging between 2 and about 20 but the optimal values vary across tests.

\author{
[ Insert Figure 3 about here ]
}

The left panels of Figure 3 show the percentage of truncated observations per interval (at the 1minute frequency) over 25,000 replications (i.e. 100 times 250 days) for $a_{0}=3,6,7,8,9,10$. Truncation frequencies display spurious periodicity due to the fact that the spot volatility is incorrectly estimated at a constant level throughout the day.

To correct this inappropriate periodicity in truncation, we propose to filter out the intraday periodicity prior to truncating, where filtered returns are defined as $r_{t, i}^{*}=r_{t, i} / \hat{f}_{t, i}$ and $\hat{f}_{t, i}$ estimates the periodic component of the volatility obtained using a modified version of the WSD estimator of Boudt et al. (2011b) (see Appendix B for more details). The right panels of Figure 3 show that this filter eliminates the inappropriate periodicity in truncation. The next section examines the effectiveness of this approach.

\title{
4.3. Simulation results: size and power
}

To determine the size and power for the tests presented in Section 2.2, we apply them to data simulated from the 3 data generating processes described in Section 4.1. We set the sampling frequency $\Delta$ to 30 -seconds and therefore generate 2,880 observations per day. ${ }^{11}$

\subsection{Results for the Brownian motion (BM) test}

We start by presenting the results for the Brownian motion (BM) test (i.e. hypothesis (A)). To study the size of this test in presence of intraday periodicity, we simulate data from DGP1 and apply the test for BM. Table 1 reports the rejection frequencies of the test applied on raw data (denoted BM test) and on filtered data (denoted BM-WSD test). We consider 6 values of $a_{0}$, i.e., $6,7,8,10,12$ and 15.

\section{[ Insert Table 1 about here ]}

Table 1 indicates that periodicity has strong effects on the size of the uncorrected BM tests. The test applied on raw data (labeled "BM test") is undersized for $a_{0}=6,7$ and 8 which corresponds (see Figure 3) to the cases where too many observations were truncated when periodicity is very high.

\footnotetext{
${ }^{11}$ The tests are applied at the $1 \%, 5 \%$ and $10 \%$ significance levels. Recall that all tests are one-tailed tests. In particular, test (C) is a right-tailed test while the others are left-tailed tests.
} 
Unlike the uncorrected test, the BM-WSD test is correctly sized and insensitive at all to the choice of $a_{0}$ for the range of values proposed by Aït-Sahalia and Jacod (2012) (ASJ).

\subsection{Results for the jump test}

To assess the accuracy of the jump tests, we compare the rejection frequencies of the periodicitycorrected test (using WSD) with that of the uncorrected tests. We consider the tests of the null hypotheses of "no jumps" (B) and "jumps" (C), respectively. Tables 2 and 3 report the rejection frequencies of the jump tests under DGP1 and DGP2, respectively. These two tables are split into two parts because the ranges of $a_{0}$ values advocated by ASJ differ for the two tests. For the test of $H_{0}$ : Jumps, we chose $a_{0}=3,4$ and 5 while for the test of $H_{0}$ : NoJumps, we use the same values than for the other tests, i.e., $a_{0}$ between 6 and 15 .

[ Insert Tables 2 and 3 about here ]

Under DGP1, there are no jumps and therefore the DGP satisfies the null hypothesis $(\mathbf{B}) H_{0}$ : NoJumps but does not satisfy the null hypothesis (C) $H_{0}$ : Jumps. Rejection frequencies in the top panel of Table 2 show the power of the test while those in the lower panel of the table show the size. Table 2 indicates that both the original test and the corrected test (with WSD) have good size and power. The top panel of the table shows that the tests nearly always correctly reject the null of jumps. The lower panel of Table 2 shows that under the null hypothesis of no jumps (i.e., $H_{0}$ : NoJumps), the tests are nearly correctly sized. The test applied on filtered returns, denoted $H_{0}$ : NoJumps-WSD, is slightly less undersized.

Table 3 reports the rejection frequencies when returns follow DGP2 (i.e., a GARCH process with finite-activity jumps). In this case, the top panel of the table shows that the original test (i.e. $H_{0}$ : Jumps) is highly oversized at all levels and for all threshold $\left(a_{0}\right)$ values. However, the test applied to filtered returns is approximately correctly sized. For example, the rejection frequencies of the original test are about $23 \%$ and $29 \%$ (at the $5 \%$ and $10 \%$ nominal levels, respectively) while they are about $6 \%$ and $11 \%$, respectively, for the test applied on filtered returns. Moreover, the corrected test is always more powerful than the uncorrected test and this difference can be very substantial for high values of $a_{0}$ (e.g. $80 \%$ vs. $99 \%$ for $\left.a_{0}=15\right)$.

\subsection{Results for the finite activity (FA) and infinite activity (IA) tests}

We now focus on the size and power of the tests for jump activity. As previously, we consider two null hypothesis: (D) jumps have finite-activity (i.e., $H_{0}$ : FA), and (E) jumps have infinite-activity (i.e., $H_{0}$ : IA). Tables $4-6$ report the rejection rates under DGP1, DGP2 and DGP3, respectively. 


\section{[ Insert Tables $4-6$ about here ]}

The null hypothesis $H_{0}$ : FA is satisfied under DGP1 and DGP2 and therefore rejection frequencies reported in Tables 4 and 5 for this test are sizes. Interestingly, the uncorrected test is very oversized for $a_{0}<10$ for both DGP1 and DGP2 while the corrected test has appropriate size for any choice of $a_{0}$ in the range suggested by Aït-Sahalia and Jacod (2011).

Tables 4 and 5 also report rejection frequencies (i.e., power) for the test of infinite activity $\left(H_{0}\right.$ : IA). Table 4 shows that both the corrected and uncorrected tests for infinite activity (H0: IA) are very powerful against the null of finite activity. In contrast, Table 5 shows that the corrected (i.e., WSD filtered) tests are more powerful than uncorrected tests against the IA null, especially for higher values of $a_{0}$. The power improvement can be very substantial e.g., $68 \%$ vs. $100 \%$ for $a_{0}=15$ for a nominal size of $5 \%$.

Recall that DGP3 exhibits IA jumps and therefore rejection rates for the null of IA represent size while rejection rates for the null of FA represent power. With this in mind, we observe that Table 6 indicates that both tests for IA tend to be slightly oversized. However, both corrected and uncorrected tests exhibit good power against the null of finite activity, with rejection rates often nearing $100 \%$.

In summary, the simulation analysis reveals three main findings. First, the periodicity does not have a strong impact on BM tests. Our proposed BM-WSD test is correctly sized regardless of $a_{0}$, whereas the original test is undersized for low $a_{0}$ values.

Second, our WSD-filtered jump test has better size under the null of jumps and more power against the null of no jumps than the uncorrected test. Under a jump-diffusion process (i.e., DGP2), for instance, the original FA test is oversized for low values of $a_{0}$ but marginally undersized for high values of $a_{0}$ (Table 5). For the same data generating process (i.e., DGP2), the power of the uncorrected IA test falls off markedly as $a_{0}$ increases. The size distortion of the original test is relatively high, and it does not diminish for different $a_{0}$ values. The size of our proposed test is correct and not sensitive to the threshold, $a_{0}$.

Finally, Tables 4 and 5 show that the periodicity-filtered FA test has better size (when $H_{0}$ : FAWSD) and more power (when $H_{0}$ : IA-WSD) than the uncorrected test. Under DGP2, the original FA test is oversized for small threshold values, and undersized for large threshold values. For the same DGP, the original IA test loses substantial power for large $a_{0}$, whereas our activity tests behave well. 


\section{Empirical analysis}

This section reports the results of the tests for the 1-minute EUR/USD, USD/JPY, and GBP/USD returns. We apply the uncorrected and corrected tests for BM, jumps, no-jumps, FA jumps and IA jumps to each day of real data and report the proportion of days in which we reject the null hypothesis.

[ Insert Table 7 about here ]

The first two rows in Table 7 provide strong evidence of BM in the data. At the 1 percent significance level, the corrected BM-WSD test never rejects the null of BM for any exchange rate. The rejection rates are much smaller than the nominal sizes of the tests-1\% and 5\%-considered in Table 7. However, the asymptotic distribution of this test statistic has been derived under the assumption of noiseless prices. Market microstructure noise will skew the tests' finite sample distribution to the right, away from rejction (i.e., $S_{W}=k$ belongs to the non rejection region of the test). Consequently, the presence of noise is likely to bias the test away from rejecting BM.

Having established evidence for BM, we now test for jumps at the daily frequency. As in Aït-Sahalia and Jacod (2009b), we consider both null hypotheses of jumps and no-jumps. The fourth and sixth rows of Tables 7 report the rejection frequencies of the periodicity-corrected test under two hypotheses "jumps" and "no-jumps", respectively. At the $1 \%$ significance level, the corrected tests reject the null of jumps on 30 to $37 \%$ of days, depending on the exchange rate, indicating that at least $1 / 3$ of days do not have jumps (row 4). Similarly, the null hypothesis of no-jumps is rejected in 27 to $29 \%$ of the time, at the 1 percent level, providing strong evidence that this $\%$ of days do have jumps (row 6 ). Of course, statistical tests do not have perfect power against alternatives so we can reject neither hypothesis on $38 \%$ of days.

Corrected tests reject the null of jumps less often than uncorrected tests while they reject the null of no-jumps less often. This is consistent with our findings in the previous section that the uncorrected test for jumps rejects the null of jumps much more often on simulated data with jumps and intraday periodicity in volatility leading to a strong size distortion. Likewise, corrected tests reject the null of jumps less often than uncorrected tests. That is, under either null, the corrected tests find more support for jumps.

We can examine the type of jumps - large and rare versus small and frequent - with FA and IA jump tests. The 8 th row of Tables 7 shows that the corrected test rejects the null of FA jumps for 36 to 45 percent of the days at the 1 percent significance level, in favor of the alternative of IA jumps or just BM. Similarly, the corrected tests reject IA jumps 69 to $73 \%$ of the time at the $1 \%$ level, in favor 
of FA jumps or BM. The results provide evidence of both finite and infinite jump activity in the data but the tests reject IA jumps much more often than FA, indicating stronger support for FA jumps. It is very possible - even likely - however, that IA behavior best describes many days.

\subsection{Time-varying rejection rates}

Forex trading changed substantially during our 2000-2010 sample with the use of electronic trading and high frequency algorithms expanding greatly. These changes in trading as well as episodes such as the financial crisis of 2008-2009 could easily generate time variation in statistical properties. To investigate time variation in rejection rates for the tests-jumps, no-jumps, FA and IA — we fit a probit model to the daily series of $1 \%$ level rejection indicators $(0,1)$ for each of the four tests conducted on each of the three exchange rates. The very low rejection rates for the test of BM precluded time variation. To explain time variation in rejections, we used a sixth order polynomial of time in a probit model. The probit regression coefficients were often statistically significant for even the sixth order polynomialsresults omitted for brevity - and therefore there was statistically significant time variation in nearly all the rejection series. The common model seemed to fit all the series fairly well. ${ }^{12}$

\section{[ Insert Figure 4 about here ]}

The left-hand panels of Figure 4 show the fitted values for the $1 \%$ rejection rates for the tests of the nulls of jumps and no-jumps. ${ }^{13}$ Not surprisingly, the rejection rates are negatively correlated for these two tests for all three exchange rates. The most pronounced feature of these series is the increasing (decreasing) rate of rejection for jumps (no-jumps) starting in the summer of 2007 that coincides with the start of the housing crisis turmoil in financial markets.

The right-hand panels of Figure 4 likewise show the rejection rates for the nulls of FA and IA for each exchange rate. IA tests show the highest rejection rates of any test for all three series; the IA rates do not vary too much over the sample, with only 2 or 3 significant coefficients for each of the 3 regressions. The time varying rejection rates for FA and for no-jumps are positively correlated, generally trending downward over the sample and covarying positively, except during the financial crisis (2008-2009) when FA rejection rates temporarily increased but no-jump rejections continued to decline.

\footnotetext{
${ }^{12}$ The probit regressors were a constant, a time trend, a time trend squared, etc., out to a time trend to the 6 th power. For computational convenience, we orthogonalized the regressor power $N$ with respect to the regressors powers 0 through $N-1$ and then standardized all the regressors to have unit variance. There were 2 to 6 statistically significant polynomial coefficients for each of the 12 regressions, with an average of about 4 per regression. We did not attempt to tailor the regressor sets to individual rejection series as experimentation with more careful model selection did not much change the in-sample fits.

${ }^{13}$ The figure omits the first and last 6 months of the sample as it is well known that such polynomial approximations are unreliable near the borders of the sample.
} 
In summary, there is strong evidence of statistically significant time variation in these 12 rejection series and the patterns were fairly similar across exchange rates. Paradoxically, the volatile markets of the financial crisis made it easier to reject jumps and harder to reject no-jumps. The next section highlights the implications of these findings for FX market microstructure and currency option pricing.

\subsection{Discussion of the empirical results}

We discuss the implications of empirical results from two perspectives, first, by utilizing insights from the research on FX market microstructure to explain the nature of jumps in the data and second, linking our analysis to option pricing. Our discussion motivates having both FA and IA jumps for pricing options and measuring kurtosis.

\subsubsection{Why do exchange rate jumps occur so frequently? News versus price-contingent trading}

Our empirical analysis reveals strong evidence of jumps in the foreign exchange data. Row 6 of Table 7 shows that we can reject the null of no-jumps on almost $30 \%$ of days at the $1 \%$ level, suggesting that there are jumps on at least this many days. In addition, FA jumps (rare and large) can be rejected on only $35-45 \%$ of days but IA jumps (frequent and small) can be rejected $70-75 \%$ of the time, again, at the $1 \%$ level.

Although the literature has linked news events to jumps (see e.g., Dewachter et al., 2014, Lahaye et al., 2011) formal news releases explain only small proportion of jumps and news does not always produce jumps. For instance, Lahaye et al. (2011) show that the probability of observing an exchange rate jump - conditional on macro news - is around 1\%. Dewachter et al. (2014) confirm this result for policy speeches and verbal foreign exchange interventions. One explanation could be that researchers typically relate announcements to large FA jumps (e.g., Poisson jumps) that are more easily found than smaller IA jumps.

In contrast to previous results (e.g., Todorov and Tauchen, 2010, Cont and Mancini (2011)), we provide evidence that jumps have both finite and infinite activity (e.g., Poisson jumps and CGMY jumps, respectively). We interpret this finding as follows. While news announcements lead to extreme (and rare) exchange rate movements, IA jumps could be linked to continuously adjusting market microstructure dynamics, including inventory reallocation. This explanation is consistent with the view of Osler and Savaser (2011), who show that extreme FX fluctuations occur frequently and cluster over time with relatively small magnitudes. Osler (2005) and King et al. (2013) argue that price-contingent trading can increase jump frequency; significant movements trigger stop-loss orders that prompt further jumps. Thus, news and market microstructure combine to generate a self-exciting series of small jumps. 
In other words, while large FA jumps are often associated with news announcements, microstructure dynamics and price-contingent trading might explain the presence of IA jumps in exchange rates.

\subsubsection{Implications for currency option pricing}

Studies in foreign exchange option pricing considered continuous-time models with characteristics that include diffusion and jumps. For example, Borensztein and Dooley (1987) propose a pure jump model that has no BM. Jorion (1988) and Bates (1996a) challenged this view and developed a jumpdiffusion process for option prices. Further research in this strand included the works of Chesney and Scott (1989) and Melino and Turnbull (1990), who combined diffusion with stochastic volatility. Bates (1996b) extended these works to the more general environment of jump-diffusion with stochastic volatility.

Despite this substantial progress, jump fear documented in the previous research remains too low to match the excess kurtosis found in exchange rate data. Bates (1996b) addresses the excess kurtosis puzzle and shows that infrequent large jumps significantly improve the ability of an exchange rate model to fit deutsche-mark option prices. In addition, rare jump events partially explain the excess kurtosis observed in spot exchange rates.

Our analysis offers two potential resolutions to this issue. First, jumps probably occur more frequently than documented in the currency option pricing literature: The proportion of jump days is at least 30\%; jumps are not rare events. A model incorporating high levels of jump activity might better explain the empirical characteristics of option prices and spot forex excess kurtosis.

Second, our results provide evidence that exchange rate jumps have both FA and IA. While FA jumps are large and relatively rare, IA jumps are typically small and frequent. An option pricing model that contains both types of jumps could improve a stochastic volatility model with single type of jump. This more complex "three-component model" - (i.e., (1) stochastic volatility, (2) finite activity jumps, (3) infinite activity jumps) - is likely to better capture the empirical characteristics of exchange rate jumps.

\section{Conclusion}

This paper has studied the most appropriate features of high frequency foreign exchange rates. It makes both methodological and empirical contributions to the literature. Methodologically, we use simulations to show that intraday periodicity in volatility can badly distort the size and power of ASJ tests of BM, jumps, no-jumps, FA jumps and IA jumps. We propose a correction to remove the intraday periodicity in volatility that restores the desired test properties. 
We apply these corrected tests to more than ten years of 1-minute data on 3 exchange rates, finding fairly consistent results across the 3 exchange rates but substantial variation in rejection rates over time. We draw several conclusions: First, the test of BM almost never rejects the BM null. That is, the test must be undersized. We reason that microstructure noise probably biases the test toward rejection and that we are safe to conclude that Brownian motion characterizes the data. Second, one can reject the null hypothesis of jumps for about $1 / 3$ of days, at the one percent level, and one can reject the null of no-jumps on a slightly lower fraction of days. The data appear to include substantial fractions of days with and without jumps, as well as many days where neither hypothesis can be rejected. Third, tests of jump activity reject the null of FA jumps for 35 to 45 percent of days and the null of IA jumps for 69 to 73 percent of days. This suggests that the data are more often consistent with FA jumps but also consistent with IA jumps on many days.

The increasing use of electronic trading, algorithmic trading and the introduction of very volatile conditions during the financial crisis might have changed the properties of high frequency returns over our long 2000-2010 sample. This possibility prompted us to investigate time variation in test rejection rates. We find, seemingly paradoxically, that tests are significantly more likely to reject jumps (and less likely to reject no-jumps) after the start of the financial crisis in the summer of 2007. Although rejection rates for IA jumps exhibit statistically significant variation, the variation does not seem very important economically. In contrast, FA jump rejection rates trended down during the sample, until the volatile period of 2008-2009, when they temporarily rose again.

We conclude that a 3-component model-Brownian motion, finite and IA jumps - characterizes 1minute foreign exchange data better than a simpler 2-component jump-diffusion. We recommend that researchers in high-frequency asset prices account for intraday periodicity, the possibility of IA and time variation in the data generating process.

\section{Acknowledgments}

We thank Yacine Aït-Sahalia, Torben Andersen, Christian Conrad, Dobrislav Dobrev, Mardi Dungey, Malik Fall, Catherine Forbes, Nikola Gradojevic, Nikolaus Hautsch, Jean Jacod, Menelaos Karanasos, Marius Matei, Neil Shephard, George Tauchen, Victor Todorov, David Veredas, Michel van der Wel, and participants at the 1st Conference on Recent Developments in Financial Econometrics and Applications-Deakin University, Econometric Society-Australian Meeting, 3rd Humboldt-Copenhagen Financial Econometrics Conference, Université Catholique de Louvain CESAM Seminar, Bogazici University Annual CEE Conference, Midwest Finance Association Meeting, and IÉSEG School of Management Finance Seminar for valuable comments and helpful suggestions.

\section{Appendix A. Standardized test statistics and estimation of asymptotic variances}

In this section, we present the standardized versions of the test statistics in (5) to (8). We use these standardized versions to apply the testing procedures. The standardized version of $\widehat{S}_{W}(p, u, k, \Delta)_{t}$ is 
defined as

$$
\frac{\widehat{S}_{W}(p, u, k, \Delta)_{t}-k^{1-p / 2}}{\sqrt{\widehat{V}_{t}^{w}}},
$$

where $\widehat{V}_{t}^{w}$ is the asymptotic variance estimator of $\widehat{S}_{W}(p, u, k, \Delta)_{t}$ and is given by

$$
\widehat{V}_{t}^{w}=N(p, k) \frac{\widehat{B}(2 p, u, \Delta)_{t}}{\left(\widehat{B}(p, u, \Delta)_{t}\right)^{2}},
$$

with

$$
N(p, k)=\frac{1}{m_{2} p}\left(k^{2-p}(1+k) m_{2 p}+k^{2-p}(k-1) m_{p}^{2}-2 k^{3-3 p / 2} m_{k, p}\right),
$$

where

$$
\begin{aligned}
m_{p} & \equiv \mathbb{E}\left(|U|^{p}\right)=\frac{2^{p / 2}}{\sqrt{\pi}} \Gamma\left(\frac{p+1}{2}\right) \\
m_{k, p} & \equiv \mathbb{E}\left(|U|^{p}|U+\sqrt{k-1} V|^{p}\right)=\frac{2 p}{\sqrt{\pi}}(k-1)^{p / 2} \Gamma\left(\frac{1+r}{2}\right)^{2} F_{2,1}\left(-\frac{p}{2} ; \frac{p+1}{2} ; \frac{1}{2} ; \frac{-1}{k-1}\right),
\end{aligned}
$$

where $U$ and $V$ are two independent $\mathcal{N}(0,1)$ variables and $F_{2,1}$ is Gauss's hypergeometric function. Under the null hypothesis that Brownian motion is present (i.e., Hypothesis (A)), Aït-Sahalia and Jacod (2010) show that the standardized test statistic in (B.1) is asymptotically (as $\Delta \rightarrow 0$ ) standard normal.

To test the null hypothesis of jumps, the standardized version of the $\widehat{S}_{J}(p, k, \Delta)_{t}$ in $(6)$ can be defined as

$$
\frac{\widehat{S}_{J}(p, k, \Delta)_{t}-k^{p / 2-1}}{\sqrt{\widehat{V}_{t}^{c}}},
$$

where $\widehat{V}_{t}^{c}$ is the asymptotic variance estimator of $\widehat{S}_{J}(p, k, \Delta)_{t}$ under the null of hypothesis of no jumps. That is,

$$
\widehat{V}_{t}^{c}=\frac{\Delta M(p, k) \widehat{A}(2 p, u, \Delta)_{t}}{\widehat{A}(p, u, \Delta)_{t}^{2}}
$$

where

$$
\widehat{A}(p, u, \Delta):=\frac{(1 / M)^{1-p / 2}}{m_{p}} \sum_{i=1}^{1 / \Delta}\left|r_{t, i}\right|^{p} 1_{\left\{\left|r_{t, i}\right| \leq u\right\}},
$$

with

$$
M(p, k)=\frac{1}{m_{p}^{2}}\left(k^{p-2}(1+k) m_{2 p}+k^{p-2}(k-1) m_{p}^{2}-2 k^{p / 2-1} m_{k, p}\right),
$$

where the quantities $m_{p}$ and $m_{k, p}$ are defined above Aït-Sahalia and Jacod (2009b) show that the standardized test statistic in (B.4) is asymptotically (as $\Delta \rightarrow 0$ ) standard normal when there are no jumps (i.e., Hypothesis (B)).

Under the null hypothesis that jumps are present, the estimator of the asymptotic variance takes a different form. In this case,

$$
\frac{\widehat{S}_{J}(p, k, \Delta)_{t}-1}{\sqrt{\widehat{V}_{t}^{j}}},
$$

where the estimator of the asymptotic variance is

$$
\widehat{V}_{t}^{j}=\frac{\Delta(k-1) p^{2} \widehat{D}(2 p-2, u, \Delta)_{t}}{2 \widehat{B}(p, \infty, \Delta)_{t}^{2}},
$$


with

$$
\widehat{D}(p, u, \Delta):=\frac{1}{k \Delta} \sum_{i=1}^{1 / \Delta}\left|r_{t, i}\right|^{p} \sum_{j \in I_{t}(i)}\left(r_{t, i}^{j}\right)^{2} 1_{\left\{\left|r_{t, i}^{j}\right| \leq u\right\}} .
$$

The standardized version of the $\widehat{S}_{J}(p, k, \Delta)_{t}$ in (7) can be defined as

$$
\frac{\widehat{S}_{F A}(p, u, k, \Delta)_{t}-k^{p / 2-1}}{\sqrt{\widehat{V}_{t}^{F A}}},
$$

where

$$
\widehat{V}_{t}^{F A}=N(p, k) \frac{\widehat{B}(2 p, u, \Delta)_{t}}{\left(\widehat{B}(p, u, \Delta)_{t}\right)^{2}}
$$

with

$$
N(p, k)=\frac{1}{m_{2} p}\left(k^{p-2}(1+k) m_{2 p}+k^{p-2}(k-1) m_{p}^{2}-2 k^{p / 2-1} m_{k, p}\right),
$$

where $m_{p}$ and $m_{k, p}$ are defined same as above. Under the null hypothesis that jumps have finite activity (i.e., Hypothesis (D)), the standardized test statistic in (B.11) is asymptotically (as $\Delta \rightarrow 0$ ) standard normal. Finally, as we consider the null hypothesis that jumps have infinite activity (i.e., Hypothesis (E)), then the standardized version of the $\widehat{S}_{I A}\left(p, p^{\prime}, u, \gamma, \Delta\right)_{t}$ in (8) is defined as

$$
\frac{\widehat{S}_{I A}\left(p, p^{\prime}, u, \gamma, \Delta\right)_{t}-\gamma^{p^{\prime}-p}}{\sqrt{\widehat{V}_{t}^{I A}}},
$$

where the asymptotic variance $\widehat{V}_{t}^{I A}$ takes the form

$$
\begin{aligned}
\widehat{V}_{t}^{I A}=\gamma^{2 p^{\prime}-2 p} & \left(\frac{\widehat{B}(2 p, u, \Delta)_{t}}{\left(\widehat{B}(p, u, \Delta)_{t}\right)^{2}}+\left(1-2 \gamma^{-p}\right) \frac{\widehat{B}(2 p, \gamma u, \Delta)_{t}}{\left(\widehat{B}(p, \gamma u, \Delta)_{t}\right)^{2}}+\frac{\widehat{B}\left(2 p^{\prime}, u, \Delta\right)_{t}}{\left(\widehat{B}\left(p^{\prime}, u, \Delta\right)_{t}\right)^{2}}+\left(1-2 \gamma^{-p^{\prime}}\right) \frac{\widehat{B}\left(2 p^{\prime}, \gamma u, \Delta\right)_{t}}{\left(\widehat{B}\left(p^{\prime}, \gamma u, \Delta\right)_{t}\right)^{2}}\right. \\
& -2 \frac{\widehat{B}\left(p+p^{\prime}, u, \Delta\right)_{t}}{\widehat{B}(p, u, \Delta)_{t} \widehat{B}\left(p^{\prime}, u, \Delta\right)_{t}}-2\left(1-\gamma^{-p}-\gamma^{-p^{\prime}}\right) \frac{\widehat{B}\left(p+p^{\prime}, \gamma u, \Delta\right)_{t}}{\left.\widehat{B}(p, \gamma u, \Delta)_{t} \widehat{B}\left(p^{\prime}, \gamma u, \Delta\right)_{t}\right) .} \quad \text { (B.16) }
\end{aligned}
$$

Under the null hypothesis that jumps have infinite activity (i.e., Hypothesis (E)), the standardized test statistic in (B.14) is asymptotically (as $\Delta \rightarrow 0$ ) standard normal.

\section{Appendix B. Volatility dynamics and periodicity estimation}

Let us consider a BSM as defined in DGP1. If $\Delta$ is sufficiently small but large enough to ensure that the efficient $\log$ prices are observed, $r_{t, i}$ is conditionally normal with mean zero and variance equal to the integral of underlying volatility over the $i$-th interval of day $t$, i.e.,

$$
\sigma_{t, i}^{2}=\int_{t+(i-1) \Delta}^{t+i \Delta} \sigma^{2}(s) d s,
$$

that is $r_{t, i} \approx \sigma_{t, i} z_{t, i}$, where $z_{t, i} \sim N(0,1)$. We assume that the high-frequency return variance $\sigma_{t, i}^{2}$ in (B.17) has a periodic component $f_{t, i}^{2}$ which represents the intraday periodic features. That is,

$$
\sigma_{t, i}=s_{t, i} f_{t, i}
$$


where $s_{t, i}$ is the stochastic intradaily volatility, constant over the day but varying from one day to another. ${ }^{14}$ The periodic factor, $f_{t, i}$, is a deterministic function of time within a day. One can estimate $s_{t, i}$ using the square root $1 / M$ times the realized variance of day $t$, i.e.,

$$
\hat{s}_{t, i}=\sqrt{\frac{1}{M} \widehat{B}(2, \infty, \Delta)_{t}} .
$$

In the presence of finite activity jumps (e.g., BSMFAJ model), (B.19) is not adequate because realized variance is not a robust estimator of the integrated variance. Therefore, Boudt et al. (2011b) recommend the use of the bipower variation estimator of Barndorff-Nielsen and Shephard (2004)'s bipower variation, which leads to the following estimate of $s_{t, i}$ :

$$
\hat{s}_{t, i}=\sqrt{\frac{1}{M-1} B V_{t}},
$$

with

$$
B V_{t} \equiv \mu_{1}^{-2} \frac{M}{(M-1)} \sum_{i=2}^{M}\left|r_{t, i}\right|\left|r_{t, i-1}\right|,
$$

where $\mu_{1} \equiv \sqrt{2 / \pi} \simeq 0.79788$. In the presence of infinite activity jumps (e.g., BSMIAJ model), we advocate the use of the threshold power variation $\left(T V_{t}\right)$ instead of $B V_{t}$ to estimate the diffusion variance, i.e.,

$$
T V_{t} \equiv \sum_{i=1}^{M}\left(r_{t, i}\right)^{2} 1_{\left|r_{t, i}\right| \leq u}
$$

where we set $u=a_{0} \sqrt{I V_{t}} \Delta^{0.5}$ and consider the $a_{0}$ values in simulations of each test. Using an appropriate estimate of $s_{t, i}$, the standardized high-frequency return $\bar{r}_{t, i}=r_{t, i} / \hat{s}_{t, i} \sim N\left(0, f_{t, i}^{2}\right)$ as $\Delta \rightarrow 0$. Boudt et al. (2011b) Boudt et al. (2011b) show that $f_{t, i}$ can therefore be obtained by estimating the scale of the standardized returns $\bar{r}_{t, i}$. Boudt et al. (2011b) recommend the use of the Shortest Half scale estimatorproposed by Rousseeuw and Leroy (1988) - because this estimator remains consistent in the presence of infinitesimal contaminations by jumps in the data. To define the Shortest Half scale estimator, we denote the corresponding order statistics $\bar{r}_{(1) ; t, i}, \ldots, \bar{r}_{\left(n_{t, i}\right) ;, i}$ such that $\bar{r}_{(1) ; t, i} \leq \bar{r}_{(2) ; t, i} \leq \ldots \leq \bar{r}_{\left(n_{t, i}\right) ; t, i}$. The shortest half scale is the smallest length of all "halves" consisting of $h_{t, i}=\left\lfloor n_{t, i} / 2\right\rfloor+1$ contiguous order observations. These halves equal $\left\{\bar{r}_{(1) ; t, i}, \ldots, \bar{r}_{\left(h_{t, i}\right) ; t, i}\right\}, \ldots,\left\{\bar{r}_{\left(n_{t, i}-h_{t, i}+1\right) ; t, i}, \ldots, \bar{r}_{\left(n_{t, i}\right) ; t, i}\right\}$, and their length is $\bar{r}_{\left(h_{t, i}\right) ; t, i}-\bar{r}_{(1) ; t, i}, \ldots, \bar{r}_{\left(n_{t, i}\right) ; t, i}-\bar{r}_{\left(h_{t, i}\right) ; t, i}$, respectively. The corresponding scale estimator (corrected for consistency under normality) equals the minimum of these lengths:

$$
\operatorname{ShortH}_{t, i}=0.741 \cdot \min \left\{\bar{r}_{\left(h_{t, i}\right) ; t, i}-\bar{r}_{(1) ; t, i}, \ldots, \bar{r}_{\left(n_{t, i}\right) ; t, i}-\bar{r}_{\left(n_{t, i}-h_{t, i}+1\right) ; t, i}\right\} \text {. }
$$

The Shortest Half estimator for the periodicity factor of $r_{t, i}$ equals

$$
\hat{f}_{t, i}^{\mathrm{ShortH}}=\frac{\text { ShortH }_{t, i}}{\sqrt{\frac{1}{M} \sum_{j=1}^{M} \text { ShortH }_{t, j}^{2}}} .
$$

The shortest half dispersion is highly robust to jumps, but it has only a $37 \%$ relative efficiency under normality of the $\bar{r}_{t, i}$ 's. Boudt et al. (2011b) show that the standard deviation applied to the returns weighted by their outlyingness under the ShortH estimate offers a better trade-off between the efficiency

\footnotetext{
${ }^{14}$ See e.g., Andersen and Bollerslev (1998a), Hecq et al. (2011), and Visser (2010) who also assume that $s_{t, i}$ is constant over the day but can vary from day to another.
} 
of the standard deviation under normality and robustness to jumps. That is,

$$
\hat{f}_{t, i}^{\mathrm{WSD}}=\frac{\mathrm{WSD}_{t, i}}{\sqrt{\frac{1}{M} \sum_{j=1}^{M} \mathrm{WSD}_{t, j}^{2}}}
$$

where

$$
\mathrm{WSD}_{t, j}=\sqrt{1.081 \cdot \frac{\sum_{l=1}^{n_{t, j}} w\left[\left(\bar{r}_{l ; t, j} / \hat{f}_{t, j}^{\text {ShortH }}\right)^{2}\right] \bar{r}_{l ; t, j}^{2}}{\sum_{l=1}^{n_{t, j}} w\left[\left(\bar{r}_{l ; t, j} / \hat{f}_{t, j}^{\text {ShortH }}\right)^{2}\right]}}
$$

Because the weighting is applied to the squared standardized returns, which are extremely large in the presence of jumps, Boudt et al. (2011b) recommend the use of the hard rejection with threshold equal to the $99 \%$ quantile of the $\chi^{2}$ distribution with one degree of freedom, that is

$$
w(z)= \begin{cases}1 & \text { if } z \leq 6.635 \\ 0 & \text { else }\end{cases}
$$

The factor 1.081 in Equation (B.26) further ensures the consistency of the estimator under normality. The Weighted Standard Deviation (WSD) in (B.25) has a $69 \%$ efficiency under normality of the $\bar{r}_{t, i}$ 's.

\section{References}

Aït-Sahalia, Y., 2004. Disentangling diffusion from jumps. Journal of Financial Economics 74, 487-528.

Ait-Sahalia, Y., Jacod, J., 2009a. Estimating the degree of activity of jumps in high-frequency data. Annals of Statistics 37, 2202-2244.

Aït-Sahalia, Y., Jacod, J., 2009b. Testing for jumps in a discretely observed process. Annals of Statistics $37,184-222$.

Aï-Sahalia, Y., Jacod, J., 2010. Is Brownian motion necessary to model high-frequency data? Annals of Statistics 38, 3093-3128.

Aït-Sahalia, Y., Jacod, J., 2011. Testing whether jumps have finite or infinite activity. Annals of Statistics 39, 1689-1719.

Aït-Sahalia, Y., Jacod, J., 2012. Analyzing the spectrum of asset returns: Jump and volatility components in high frequency data. Journal of Economic Literature 50, 1007-1050.

Aït-Sahalia, Y., Jacod, J., Li, J., 2012. Testing for jumps in noisy high frequency data. Journal of Econometrics 168, 207-222.

Andersen, T.G., Bollerslev, T., 1997. Intraday periodicity and volatility persistence in financial markets. The Journal of Empirical Finance 4, pp. 115-158.

Andersen, T.G., Bollerslev, T., 1998a. Answering the skeptics: Yes, standard volatility models do provide accurate forecasts. International Economic Review 39, 885-905.

Andersen, T.G., Bollerslev, T., 1998b. Dm-dollar volatility: Intraday activity patterns, macroeconomic announcements and longer run dependencies. The Journal of Finance 53, 219-265.

Andersen, T.G., Bollerslev, T., Diebold, F.X., 2007a. Roughing it up: Including jump components in the measurement, modelling and forecasting of return volatility. The Review of Economics and Statistics 89, 701-720.

Andersen, T.G., Bollerslev, T., Dobrev, D., 2007b. No-arbitrage semi-martingale restrictions for continuous-time volatility models subject to leverage effects, jumps and i.i.d. noise: Theory and testable distributional implications. Journal of Econometrics 138, 125-180. 
Bachelier, L., 1900. Théorie de la Spéculation. Paris: Gauthier-Villars.

Back, K., 1991. Asset prices for general processes. Journal of Mathematical Economics 20, 317-395.

Baillie, R.T., Bollerslev, T., 1991. Intra-day and inter-market volatility in foreign exchange rates. Review of Economic Studies 58, 565-585.

Bakshi, G., Carr, P., Wu, L., 2008. Stochastic risk premiums, stochastic skewness in currency options, and stochastic discount factors in international economies. Journal of Financial Economics 87, 132156.

Barndorff-Nielsen, O., Shephard, N., 2004. Power and bipower variation with stochastic volatility and jumps (with discussion). Journal of Financial Econometrics 2, 1-48.

Barndorff-Nielsen, O., Shephard, N., 2006. Econometrics of testing for jumps in financial economics using bipower variation. Journal of Financial Econometrics 4, 1-30.

Bates, D.S., 1996a. Dollar jump fears: 1984-1992: Distributional abnormalities implicit in currency future options. Journal of International Money and Finance 15, 65-93.

Bates, D.S., 1996b. Jumps and stochastic volatility: exchange rate processes implicit in deutsche mark options. Review of Financial Studies 9, 69-107.

Bollerslev, T., Todorov, V., 2011a. Estimation of jump tails. Econometrica 79, 1727-1783.

Bollerslev, T., Todorov, V., 2011b. Tails, fears and risk premia. Journal of Finance 66, 2165-2211.

Borensztein, E.R., Dooley, M.P., 1987. Options on foreign exchange and exchange rate expectations. IMF Staff Papers 34, 642-680.

Boudt, K., Croux, C., Laurent, S., 2011a. Outlyingness weighted quadratic covariation. Journal of Financial Econometrics 9, 657-684.

Boudt, K., Croux, C., Laurent, S., 2011b. Robust estimation of intraweek periodicity in volatility and jump detection. Journal of Empirical Finance 18, 353-367.

Carr, P., Geman, H., Madan, D.B., Yor, M., 2002. The fine structure of asset returns: An empirical investigation. Journal of Business 75, 305-332.

Carr, P., Madan, D.B., 1998. Option valuation using the fast fourier transform. Journal of Computational Finance 2, 61-73.

Carr, P., Wu, L., 2003. What type of process underlies options? a simple robust test. Journal of Finance 58, 2581-2610.

Carr, P., Wu, L., 2004. Time-changed Lévy processes and option pricing. Journal of Financial Economics $71,113-141$.

Carr, P., Wu, L., 2007. Stochastic skew in currency options. Journal of Financial Economics 86, $213-247$.

Chesney, M., Scott, L.O., 1989. Pricing European currency options: a comparison of the modified Black-Scholes model and a random variance model. Journal of Financial and Quantitative Analysis $24,267-284$.

Cont, R., Mancini, C., 2011. Nonparametric tests for pathwise properties of semimartingales. Bernoulli $17,781-813$.

Cont, R., Tankov, P., 2004. Financial Modelling with Jump Processes. Chapman \& Hall/CRC Financial Mathematics Series, London. 
Dewachter, H., Erdemlioglu, D., Gnabo, J.Y., Lecourt, C., 2014. The intra-day impact of communication on euro-dollar volatility and jumps. Journal of International Money and Finance 43, 131-154.

Dominquez, K.M., Panthaki, F., 2006. What defines "news" in foreign exchange markets? Journal of International Money and Finance 25, 168-198.

Drechsler, I., Yaron, A., 2011. What's vol got to do with it? Review of Financial Studies 24, 1-45.

Dungey, M., Hvozdyk, L., 2012. Cojumping: Evidence from the US Treasury bond and futures markets. Journal of Banking and Finance 36, 1563-1575.

Dungey, M., McKenzie, M., Smith, L.V., 2009. Empirical evidence on jumps in the term structure of the US Treasury market. Journal of Empirical Finance 16, 430-445.

Geman, H., 2002. Pure jump Lévy processes for asset price modelling. Journal of Banking and Finance $26,1297-1316$.

Hautsch, N., Hess, D., Veredas, D., 2011. The impact of macroeconomic news on quote adjustments, noise, and informational volatility. Journal of Banking and Finance 35, 2733-2746.

Hecq, A., Laurent, S., Palm, F., 2011. Common intraday periodicity. Forthcoming in Journal of Financial Econometrics.

Huang, J.Z., Wu, L., 2004. Specification analysis of option pricing models based on time-changed Lévy processes. Journal of Finance 59, 1405-1439.

Jorion, P., 1988. On jump processes in the foreign exchange and stock markets. Review of Financial Studies 1, 427-445.

King, M., Osler, C., Dagfinn, R., 2013. The market microstructure approach to foreign exchange: Looking back and looking forward. Journal of International Money and Finance 38, 95-119.

Lahaye, J., Laurent, S., Neely, C.J., 2011. Jumps, cojumps and macro announcements. Journal of Applied Econometrics 26, 893-921.

Lee, S.S., Hannig, J., 2010. Detecting jumps from Lévy jump-diffusion processes. Journal of Financial Economics 96, 271-290.

Li, H., Wells, M.T., Yu, C.L., 2008. Bayesian analysis of return dynamics with Lévy jumps. Review of Financial Studies 21, 2345-2378.

Li, H., Wells, M.T., Yu, C.L., 2012. Return dynamics with Lévy jumps: Evidence from stock and option prices. Forthcoming in Mathematical Finance.

Lustig, H., Verdelhan, A., 2007. The cross-section of foreign currency risk premia and consumption growth risk. American Economic Review 97, 89-117.

Madan, D.B., Carr, P.P., Chang, E.E., 1998. The variance gamma process and option pricing. European Finance Review 2, 79-105.

Maheu, J.M., McCurdy, T.H., Zhao, X., 2013. Do jumps contribute to the dynamics of the equity premium. Journal of Financial Economics 110, 457-477.

Melino, A., Turnbull, S.M., 1990. Pricing foreign currency options using stochastic volatility. Journal of Econometrics 45, 239-265.

Merton, R.C., 1976. Option pricing when underlying stock returns are discontinuous. Journal of Financial Economics 3, 125-144.

Neely, C.J., 1999. Target zones and conditional volatility: the role of realignments. Journal of Empirical Finance 6, 177-192. 
Osler, C.L., 2005. Stop-loss orders and price cascades in currency markets. Journal of International Money and Finance 24, 219-241.

Osler, C.L., Savaser, T., 2011. Extreme returns: The case of currencies. Journal of Banking and Finance $35,2868-2880$.

Rousseeuw, P., Leroy, A., 1988. A robust scale estimator based on the shortest half. Statistica Neerlandica 42, 103-116.

Todorov, V., Tauchen, G., 2006. Simulation methods for Lévy-driven CARMA stochastic volatility models. Journal of Business \& Economic Statistics 24, 455-469.

Todorov, V., Tauchen, G., 2010. Activity signature functions for high-frequency data analysis. Journal of Econometrics 154, 125-138.

Visser, M.P., 2010. GARCH parameter estimation using high-frequency data. Journal of Financial Econometrics 8, 1-36. 
Table 1: Monte Carlo rejection frequencies of the Brownian motion (BM) test under DGP1

\begin{tabular}{l|llll}
\hline \hline & $H_{0}:$ BM & $1 \%$ & $5 \%$ & $10 \%$ \\
\hline$a_{0}=6$ & & & & \\
& BM test & 0.0 & 0.1 & 0.5 \\
$a_{0}=7$ & BM-WSD test & 0.6 & 4.2 & 9.0 \\
& & & & \\
$a_{0}=8$ & BM test & 0.1 & 1.4 & 3.7 \\
& BM-WSD test & 0.6 & 4.3 & 9.2 \\
$a_{0}=10$ & BM test & 0.4 & 2.9 & 6.6 \\
& BM-WSD test & 0.6 & 4.3 & 9.1 \\
& & & & \\
$a_{0}=12$ & BM test & 0.6 & 4.0 & 8.7 \\
& BM-WSD test & 0.6 & 4.4 & 9.2 \\
& BM test & 0.6 & 4.0 & 8.7 \\
$a_{0}=15$ & BM-WSD test & 0.6 & 4.2 & 9.0 \\
& & & & \\
& BM test & 0.6 & 4.1 & 8.8 \\
& BM-WSD test & 0.6 & 4.3 & 9.1 \\
\hline \hline
\end{tabular}

Notes: The table reports the rejection frequencies of the BM test and its periodicitycorrected version (BM-WSD test). The data generating process is DGP1. We set $p=1.5$ and $k=2$. Because DGP1 features BM, the numbers in this table correspond to the empirical size of the test for BM. 
Table 2: Monte Carlo rejection frequencies of the jump tests under DGP1

\begin{tabular}{|c|c|c|c|c|}
\hline & & $1 \%$ & $5 \%$ & $10 \%$ \\
\hline \multirow[t]{3}{*}{$a_{0}=3$} & & & & \\
\hline & $H_{0}:$ Jumps & 99.8 & 100.0 & 100.0 \\
\hline & $H_{0}$ : Jumps-WSD & 100.0 & 100.0 & 100.0 \\
\hline \multicolumn{5}{|l|}{$a_{0}=4$} \\
\hline & $H_{0}$ : Jumps & 99.7 & 100.0 & 100.0 \\
\hline & $H_{0}$ : Jumps-WSD & 100.0 & 100.0 & 100.0 \\
\hline \multirow[t]{3}{*}{$a_{0}=5$} & & & & \\
\hline & $H_{0}$ : Jumps & 99.7 & 100.0 & 100.0 \\
\hline & $H_{0}:$ Jumps-WSD & 100.0 & 100.0 & 100.0 \\
\hline \multirow[t]{3}{*}{$a_{0}=6$} & & & & \\
\hline & $H_{0}:$ NoJumps & 0.4 & 4.0 & 9.2 \\
\hline & $H_{0}:$ NoJumps-WSD & 0.5 & 4.2 & 9.7 \\
\hline \multirow[t]{3}{*}{$a_{0}=7$} & & & & \\
\hline & $H_{0}:$ NoJumps & 0.4 & 3.8 & 9.0 \\
\hline & $H_{0}:$ NoJumps-WSD & 0.5 & 4.2 & 9.7 \\
\hline \multirow[t]{3}{*}{$a_{0}=8$} & & & & \\
\hline & $H_{0}$ : NoJumps & 0.4 & 3.8 & 9.0 \\
\hline & $H_{0}:$ NoJumps-WSD & 0.5 & 4.2 & 9.7 \\
\hline \multirow[t]{3}{*}{$a_{0}=10$} & & & & \\
\hline & $H_{0}:$ NoJumps & 0.4 & 3.8 & 9.0 \\
\hline & $H_{0}:$ NoJumps-WSD & 0.5 & 4.2 & 9.7 \\
\hline \multirow[t]{3}{*}{$a_{0}=12$} & & & & \\
\hline & $H_{0}:$ NoJumps & 0.4 & 3.8 & 9.0 \\
\hline & $H_{0}:$ NoJumps-WSD & 0.5 & 4.2 & 9.7 \\
\hline \multirow[t]{3}{*}{$a_{0}=15$} & & & & \\
\hline & $H_{0}:$ NoJumps & 0.4 & 3.8 & 9.0 \\
\hline & $H_{0}:$ NoJumps-WSD & 0.5 & 4.2 & 9.7 \\
\hline
\end{tabular}

Notes: The table reports the rejection frequencies of the jumps test and their periodicity-corrected versions (with WSD correction). The data generating process is DGP1. We set $p=4$ and $k=2$. Because DGP 1 features no jumps, the numbers in the upper (lower) panels correspond to the empirical power (size). 
Table 3: Monte Carlo rejection frequencies of the jump tests under DGP2

\begin{tabular}{|c|c|c|c|c|}
\hline & & $1 \%$ & $5 \%$ & $10 \%$ \\
\hline \multirow[t]{3}{*}{$a_{0}=3$} & & & & \\
\hline & $H_{0}$ : Jumps & 15.1 & 23.4 & 29.4 \\
\hline & $H_{0}:$ Jumps-WSD & 1.5 & 6.2 & 11.2 \\
\hline \multicolumn{5}{|c|}{ 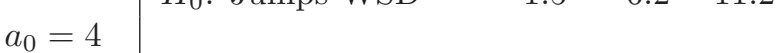 } \\
\hline & $H_{0}$ : Jumps & 14.8 & 23.0 & 28.9 \\
\hline & $H_{0}:$ Jumps-WSD & 1.5 & 6.1 & 11.2 \\
\hline \multirow[t]{3}{*}{$a_{0}=5$} & & & & \\
\hline & $H_{0}$ : Jumps & 14.7 & 22.9 & 28.8 \\
\hline & $H_{0}:$ Jumps-WSD & 1.5 & 6.1 & 11.2 \\
\hline \multirow[t]{3}{*}{$a_{0}=6$} & & & & \\
\hline & $H_{0}:$ NoJumps & 92.7 & 96.8 & 98.0 \\
\hline & $H_{0}:$ NoJumps-WSD & 99.9 & 99.9 & 99.9 \\
\hline \multirow[t]{3}{*}{$a_{0}=7$} & & & & \\
\hline & $H_{0}:$ NoJumps & 92.1 & 96.7 & 97.8 \\
\hline & $H_{0}:$ NoJumps-WSD & 99.9 & 99.9 & 99.9 \\
\hline \multirow[t]{3}{*}{$a_{0}=8$} & & & & \\
\hline & $H_{0}:$ NoJumps & 92.0 & 96.1 & 97.0 \\
\hline & $H_{0}:$ NoJumps-WSD & 99.9 & 99.9 & 99.9 \\
\hline \multirow[t]{3}{*}{$a_{0}=10$} & & & & \\
\hline & $H_{0}:$ NoJumps & 90.6 & 93.0 & 93.2 \\
\hline & $H_{0}:$ NoJumps-WSD & 99.9 & 99.9 & 99.9 \\
\hline \multirow[t]{3}{*}{$a_{0}=12$} & & & & \\
\hline & $H_{0}:$ NoJumps & 86.5 & 87.7 & 87.8 \\
\hline & $H_{0}:$ NoJumps-WSD & 99.9 & 99.9 & 99.9 \\
\hline \multirow[t]{2}{*}{$a_{0}=15$} & & & & \\
\hline & $\begin{array}{l}H_{0}: \text { NoJumps } \\
H_{0}: \text { NoJumps-WSD }\end{array}$ & $\begin{array}{l}80.1 \\
99.9\end{array}$ & $\begin{array}{l}80.9 \\
99.9\end{array}$ & $\begin{array}{l}81.0 \\
99.9\end{array}$ \\
\hline
\end{tabular}

Notes: The table reports the rejection frequencies of the jumps test and their periodicity-corrected versions (with WSD correction). The data generating process is DGP2. We set $p=4$ and $k=2$. Because DGP2 features jumps, the numbers in the upper (lower) panels correspond to the empirical size (power). 
Table 4: Monte Carlo rejection frequencies of the jump activity tests under DGP1

\begin{tabular}{|c|c|c|c|c|}
\hline & & $1 \%$ & $5 \%$ & $10 \%$ \\
\hline \multirow{3}{*}{$a_{0}=6$} & & & & \\
\hline & $H_{0}: \mathrm{FA}$ & 26.8 & 64.2 & 80.3 \\
\hline & $H_{0}:$ FA-WSD & 0.6 & 4.7 & 10.6 \\
\hline \multicolumn{5}{|l|}{$a_{0}=7$} \\
\hline & $H_{0}: \mathrm{FA}$ & 2.9 & 18.6 & 34.3 \\
\hline & $H_{0}:$ FA-WSD & 0.6 & 4.3 & 9.9 \\
\hline \multicolumn{5}{|l|}{$a_{0}=8$} \\
\hline & $H_{0}: \mathrm{FA}$ & 0.9 & 7.1 & 15.6 \\
\hline & $H_{0}:$ FA-WSD & 0.6 & 4.3 & 9.9 \\
\hline \multicolumn{5}{|l|}{$a_{0}=10$} \\
\hline & $H_{0}: \mathrm{FA}$ & 0.4 & 4.1 & 9.6 \\
\hline & $H_{0}:$ FA-WSD & 0.6 & 4.3 & 9.9 \\
\hline \multicolumn{5}{|l|}{$a_{0}=12$} \\
\hline & $H_{0}: \mathrm{FA}$ & 0.4 & 3.9 & 9.2 \\
\hline & $H_{0}:$ FA-WSD & 0.6 & 4.3 & 9.9 \\
\hline \multirow[t]{3}{*}{$a_{0}=15$} & & & & \\
\hline & $H_{0}: \mathrm{FA}$ & 0.4 & 3.9 & 9.1 \\
\hline & $H_{0}:$ FA-WSD & 0.6 & 4.3 & 9.9 \\
\hline \multirow[t]{3}{*}{$a_{0}=6$} & & & & \\
\hline & $H_{0}:$ IA & 99.9 & 100.0 & 100.0 \\
\hline & $H_{0}:$ IA-WSD & 100.0 & 100.0 & 100.0 \\
\hline \multirow[t]{3}{*}{$a_{0}=7$} & & & & \\
\hline & $H_{0}:$ IA & 99.9 & 100.0 & 100.0 \\
\hline & $H_{0}: \mathrm{IA}-\mathrm{WSD}$ & 100.0 & 100.0 & 100.0 \\
\hline \multirow[t]{3}{*}{$a_{0}=8$} & & & & \\
\hline & $H_{0}:$ IA & 99.9 & 100.0 & 100.0 \\
\hline & $H_{0}:$ IA-WSD & 100.0 & 100.0 & 100.0 \\
\hline \multirow[t]{3}{*}{$a_{0}=10$} & & & & \\
\hline & $H_{0}:$ IA & 99.9 & 100.0 & 100.0 \\
\hline & $H_{0}:$ IA-WSD & 100.0 & 100.0 & 100.0 \\
\hline \multirow[t]{3}{*}{$a_{0}=12$} & & & & \\
\hline & $H_{0}:$ IA & 99.9 & 100.0 & 100.0 \\
\hline & $H_{0}:$ IA-WSD & 100.0 & 100.0 & 100.0 \\
\hline \multirow{2}{*}{$a_{0}=15$} & $H_{0} \cdot \mathrm{I}$ & 000 & & \\
\hline & $H_{0}:$ IA-WSD & 100.0 & 100.0 & 100.0 \\
\hline
\end{tabular}

Notes: The table reports the rejection frequencies of the jump activity tests and their periodicitycorrected versions (with WSD correction). The data generating process is DGP1. We set $p=4$ and $k=2$. Because DGP1 features no jumps, the numbers in the upper (lower) panels correspond to the empirical size (power). 
Table 5: Monte Carlo rejection frequencies of the jump activity tests under DGP2

\begin{tabular}{|c|c|c|c|c|}
\hline & & $\overline{1 \%}$ & $5 \%$ & $\overline{10 \%}$ \\
\hline \multirow{3}{*}{$a_{0}=6$} & & & & \\
\hline & $H_{0}: \mathrm{FA}$ & 27.1 & 64.8 & 80.5 \\
\hline & $H_{0}:$ FA-WSD & 0.6 & 4.9 & 10.9 \\
\hline \multicolumn{5}{|l|}{$a_{0}=7$} \\
\hline & $H_{0}: \mathrm{FA}$ & 3.1 & 18.2 & 34.3 \\
\hline & $H_{0}:$ FA-WSD & 0.5 & 4.5 & 10.2 \\
\hline \multicolumn{5}{|l|}{$a_{0}=8$} \\
\hline & $H_{0}: \mathrm{FA}$ & 0.8 & 6.8 & 15.7 \\
\hline & $H_{0}:$ FA-WSD & 0.5 & 4.5 & 10.1 \\
\hline \multicolumn{5}{|l|}{$a_{0}=10$} \\
\hline & $H_{0}: \mathrm{FA}$ & 0.4 & 3.8 & 9.3 \\
\hline & $H_{0}:$ FA-WSD & 0.5 & 4.5 & 10.1 \\
\hline \multicolumn{5}{|l|}{$a_{0}=12$} \\
\hline & $H_{0}: \mathrm{FA}$ & 0.4 & 3.5 & 8.6 \\
\hline & $H_{0}:$ FA-WSD & 0.5 & 4.5 & 10.1 \\
\hline \multirow[t]{3}{*}{$a_{0}=15$} & & & & \\
\hline & $H_{0}: \mathrm{FA}$ & 0.4 & 3.3 & 8.1 \\
\hline & $H_{0}:$ FA-WSD & 0.5 & 4.5 & 10.1 \\
\hline \multirow[t]{3}{*}{$a_{0}=6$} & & & & \\
\hline & $H_{0}:$ IA & 95.3 & 96.2 & 97.0 \\
\hline & $H_{0}:$ IA-WSD & 100.0 & 100.0 & 100.0 \\
\hline \multirow[t]{3}{*}{$a_{0}=7$} & & & & \\
\hline & $H_{0}:$ IA & 91.4 & 92.4 & 93.1 \\
\hline & $H_{0}:$ IA-WSD & 100.0 & 100.0 & 100.0 \\
\hline \multirow[t]{3}{*}{$a_{0}=8$} & & & & \\
\hline & $H_{0}:$ IA & 87.0 & 88.0 & 88.7 \\
\hline & $H_{0}:$ IA-WSD & 100.0 & 100.0 & 100.0 \\
\hline \multirow[t]{3}{*}{$a_{0}=10$} & & & & \\
\hline & $H_{0}: \mathrm{IA}$ & 80.2 & 81.6 & 82.2 \\
\hline & $H_{0}:$ IA-WSD & 100.0 & 100.0 & 100.0 \\
\hline \multirow[t]{3}{*}{$a_{0}=12$} & & & & \\
\hline & $H_{0}:$ IA & 74.5 & 76.0 & 77.7 \\
\hline & $H_{0}:$ IA-WSD & 100.0 & 100.0 & 100.0 \\
\hline \multirow[t]{3}{*}{$a_{0}=15$} & & & & \\
\hline & $H_{0}:$ IA & 67.0 & 68.5 & 70.2 \\
\hline & $H_{0}:$ IA-WSD & 100.0 & 100.0 & 100.0 \\
\hline
\end{tabular}

Notes: The table reports the rejection frequencies of the jump activity tests and their periodicitycorrected versions (with WSD correction). The data generating process is DGP2. We set $p=4$ and $k=2$. Because DGP2 features FA jumps, the numbers in the upper (lower) panels correspond to the empirical size (power). 
Table 6: Monte Carlo rejection frequencies of the jump activity tests under DGP3

\begin{tabular}{|c|c|c|c|c|}
\hline & & $\overline{1 \%}$ & $5 \%$ & $10 \%$ \\
\hline \multirow{3}{*}{$a_{0}=6$} & & & & \\
\hline & $H_{0}: \mathrm{FA}$ & 83.2 & 99.9 & 100.0 \\
\hline & $H_{0}:$ FA-WSD & 84.1 & 99.9 & 100.0 \\
\hline \multicolumn{5}{|l|}{$a_{0}=7$} \\
\hline & $H_{0}: \mathrm{FA}$ & 72.8 & 99.7 & 100.0 \\
\hline & $H_{0}:$ FA-WSD & 74.2 & 99.7 & 100.0 \\
\hline \multicolumn{5}{|l|}{$a_{0}=8$} \\
\hline & $H_{0}: \mathrm{FA}$ & 62.4 & 99.5 & 100.0 \\
\hline & $H_{0}:$ FA-WSD & 64.1 & 99.5 & 100.0 \\
\hline \multicolumn{5}{|l|}{$a_{0}=10$} \\
\hline & $H_{0}: \mathrm{FA}$ & 40.3 & 98.6 & 99.9 \\
\hline & $H_{0}:$ FA-WSD & 42.5 & 98.7 & 99.9 \\
\hline \multicolumn{5}{|l|}{$a_{0}=12$} \\
\hline & $H_{0}: \mathrm{FA}$ & 19.0 & 97.3 & 99.9 \\
\hline & $H_{0}:$ FA-WSD & 20.9 & 97.6 & 99.9 \\
\hline \multirow[t]{3}{*}{$a_{0}=15$} & & & & \\
\hline & $H_{0}: \mathrm{FA}$ & 6.5 & 89.5 & 99.4 \\
\hline & $H_{0}:$ FA-WSD & 7.4 & 91.0 & 99.5 \\
\hline \multirow[t]{3}{*}{$a_{0}=6$} & & & & \\
\hline & $H_{0}:$ IA & 1.6 & 7.5 & 14.5 \\
\hline & $H_{0}:$ IA-WSD & 1.9 & 8.2 & 15.5 \\
\hline \multirow[t]{3}{*}{$a_{0}=7$} & & & & \\
\hline & $H_{0}:$ IA & 1.8 & 7.6 & 14.5 \\
\hline & $H_{0}:$ IA-WSD & 1.8 & 8.1 & 15.4 \\
\hline \multirow[t]{3}{*}{$a_{0}=8$} & & & & \\
\hline & $H_{0}:$ IA & 1.7 & 6.9 & 13.4 \\
\hline & $H_{0}:$ IA-WSD & 1.9 & 7.2 & 14.0 \\
\hline \multirow[t]{3}{*}{$a_{0}=10$} & & & & \\
\hline & $H_{0}:$ IA & 1.8 & 6.9 & 13.3 \\
\hline & $H_{0}:$ IA-WSD & 1.9 & 7.1 & 13.5 \\
\hline \multirow[t]{3}{*}{$a_{0}=12$} & & & & \\
\hline & $H_{0}: \mathrm{IA}$ & 2.0 & 6.5 & 11.9 \\
\hline & $H_{0}:$ IA-WSD & 2.0 & 6.8 & 12.8 \\
\hline \multirow[t]{3}{*}{$a_{0}=15$} & & & & \\
\hline & $H_{0}:$ IA & 2.6 & 7.9 & 13.7 \\
\hline & $H_{0}:$ IA-WSD & 2.4 & 7.7 & 14.1 \\
\hline
\end{tabular}

Notes: The table reports the rejection frequencies of the jump activity tests and their periodicitycorrected versions (with WSD correction). The data generating process is DGP3. We set $p=4$ and $k=2$. Because DGP3 features IA jumps, the numbers in the upper (lower) panels correspond to the empirical power (size). 
Table 7: Empirical results of the tests for exchange rates

\begin{tabular}{|c|c|c|c|c|c|c|}
\hline & \multicolumn{2}{|c|}{ EUR/USD } & \multicolumn{2}{|c|}{ USD/JPY } & \multicolumn{2}{|c|}{ GBP/USD } \\
\hline & $1 \%$ & $5 \%$ & $1 \%$ & $5 \%$ & $1 \%$ & $5 \%$ \\
\hline$H_{0}: \mathrm{BM}$ & 0.0 & 0.1 & 0.0 & 0.0 & 0.0 & 0.0 \\
\hline$H_{0}:$ BM-WSD & 0.0 & 0.0 & 0.0 & 0.0 & 0.0 & 0.1 \\
\hline$H_{0}$ : Jumps & 43.2 & 51.5 & 34.1 & 42.7 & 44.2 & 53.3 \\
\hline$H_{0}:$ Jumps-WSD & 35.8 & 44.2 & 30.5 & 38.2 & 36.8 & 43.5 \\
\hline$H_{0}$ : NoJumps & 17.7 & 32.9 & 24.4 & 43.2 & 21.0 & 35.1 \\
\hline$H_{0}:$ NoJumps-WSD & 28.4 & 44.1 & 29.3 & 48.7 & 27.3 & 44.4 \\
\hline$H_{0}: \mathrm{FA}$ & 28.6 & 54.0 & 43.9 & 69.4 & 31.3 & 50.4 \\
\hline$H_{0}:$ FA-WSD & 40.0 & 62.4 & 45.0 & 71.4 & 35.8 & 57.4 \\
\hline$H_{0}$ : IA & 64.7 & 77.2 & 66.0 & 77.7 & 70.3 & 81.0 \\
\hline$H_{0}:$ IA-WSD & 70.6 & 80.7 & 68.9 & 78.5 & 73.0 & 84.3 \\
\hline
\end{tabular}

Notes: The table reports the rejection frequencies (in \%) of the base and periodicity-robust tests for the EUR/USD, USD/JPY, and GBP/USD returns. The sample covers January 1, 2000 to March 12, 2010. We set $p=4$ for all test but for $H_{0}$ : BM where we set $p=1.5$. We also set $a_{0}=15$ to all test but for $H_{0}$ : Jumps where we set $a_{0}=5$. The sampling frequency is 1-minute. 


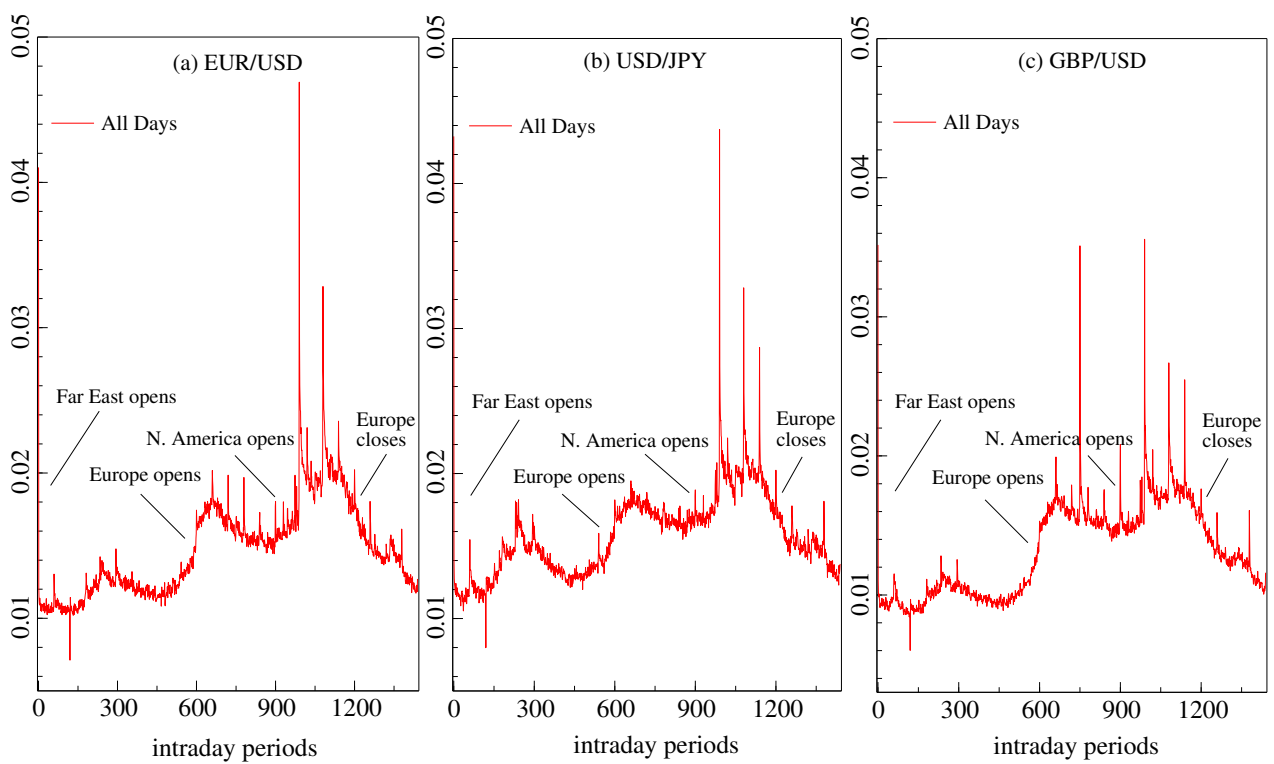

Figure 1: Mean absolute 1-min EUR/USD (left), USD/JPY (middle), and GBP/USD (right) returns on whole sample. The X-axis represents the intraday periods in EST, from 16:01 EST of day $t-1$ to 16:00 EST of day $t$. 


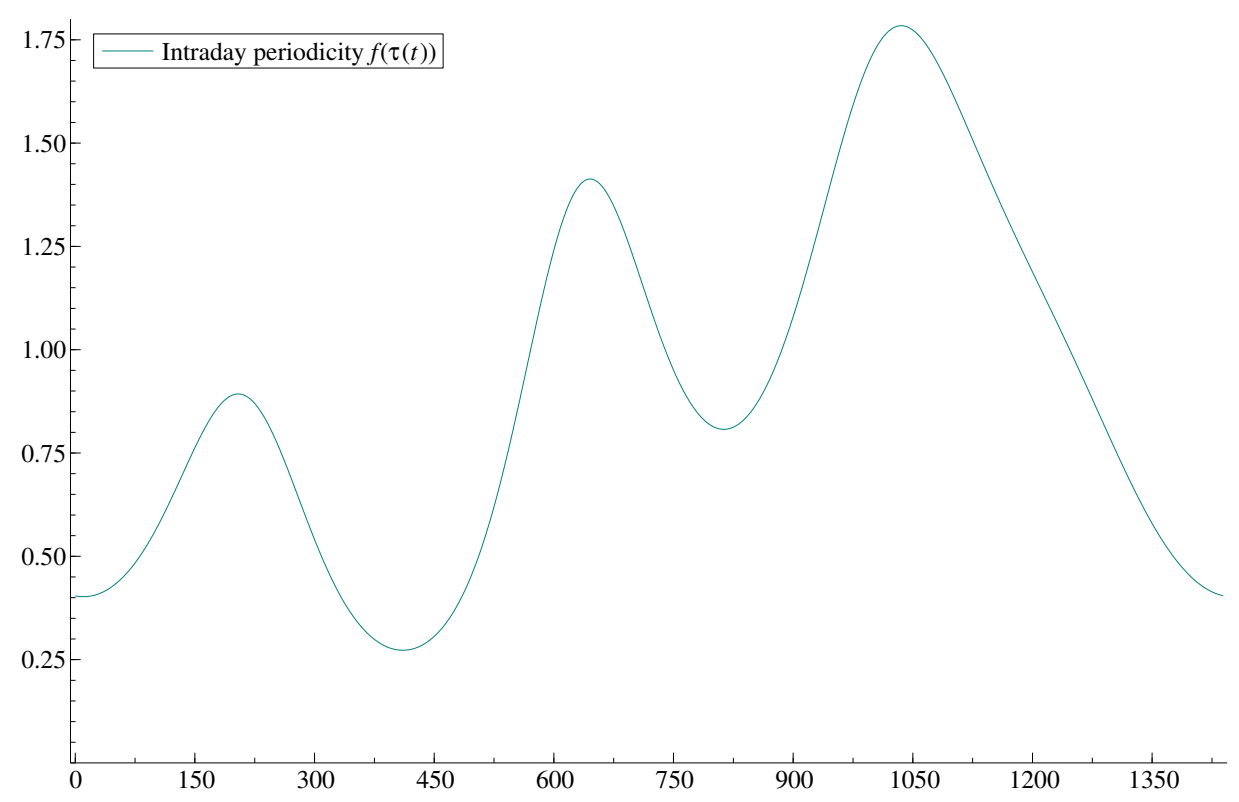

Figure 2: Simulated intraday periodicty $f(\tau(t))$
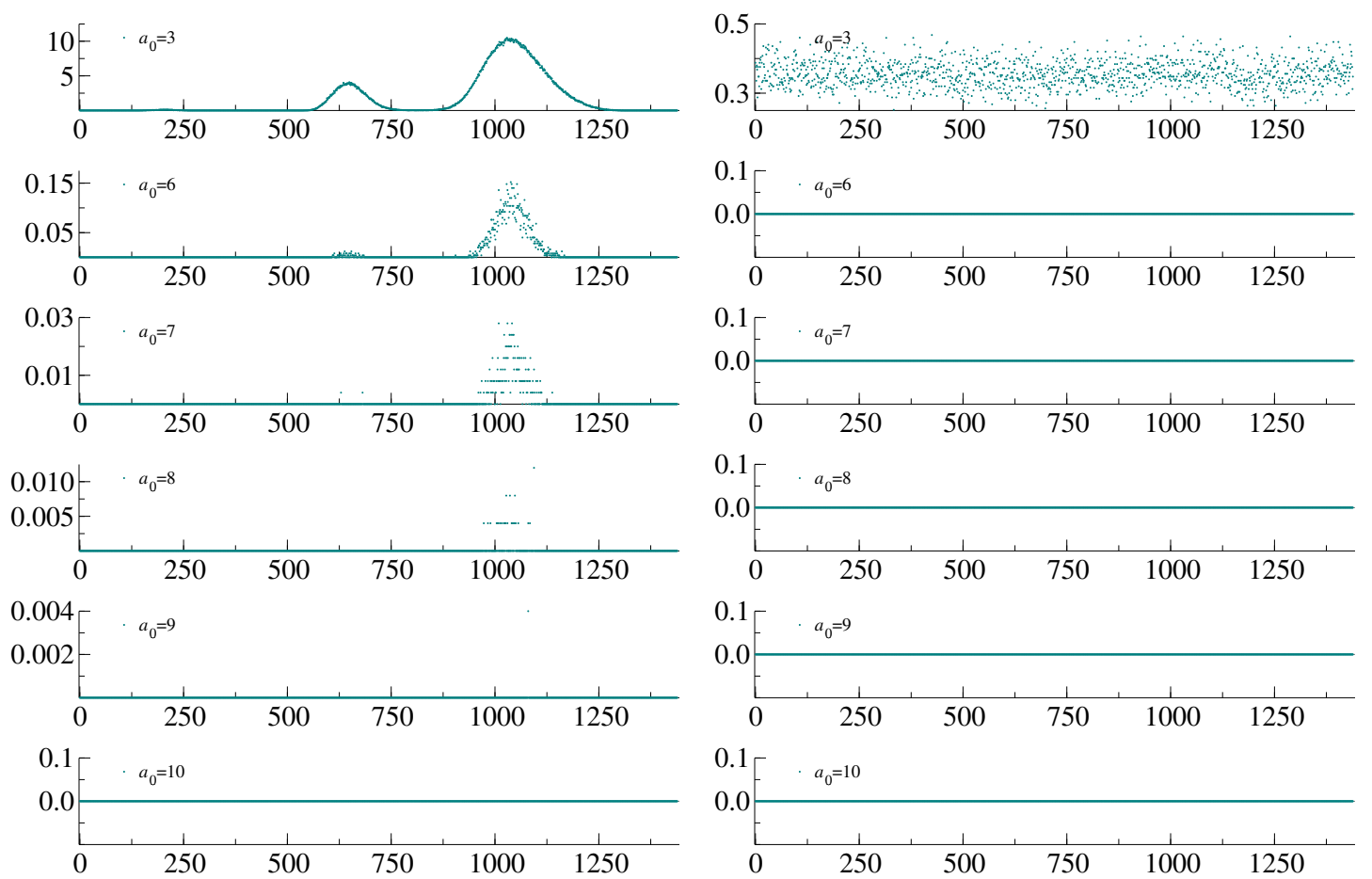

Figure 3: Frequency of truncation (in \%) per intraday interval. X-axis denotes the the intraday intervals at the 1-minute sampling frequency (i.e., $M=1,440$ ). Y-axis denotes the percentage of truncations per interval. We set the truncation threshold to $u=a_{0} \sqrt{I \hat{V}} \Delta^{0.5}$. We consider $\alpha=3,6,7,8,9,10$. The data generating process is GARCH model with periodicity. The number of replications is 25,000. Left panels: truncation without periodicity filtration. Right panels: truncation periodicity-filtered returns. 

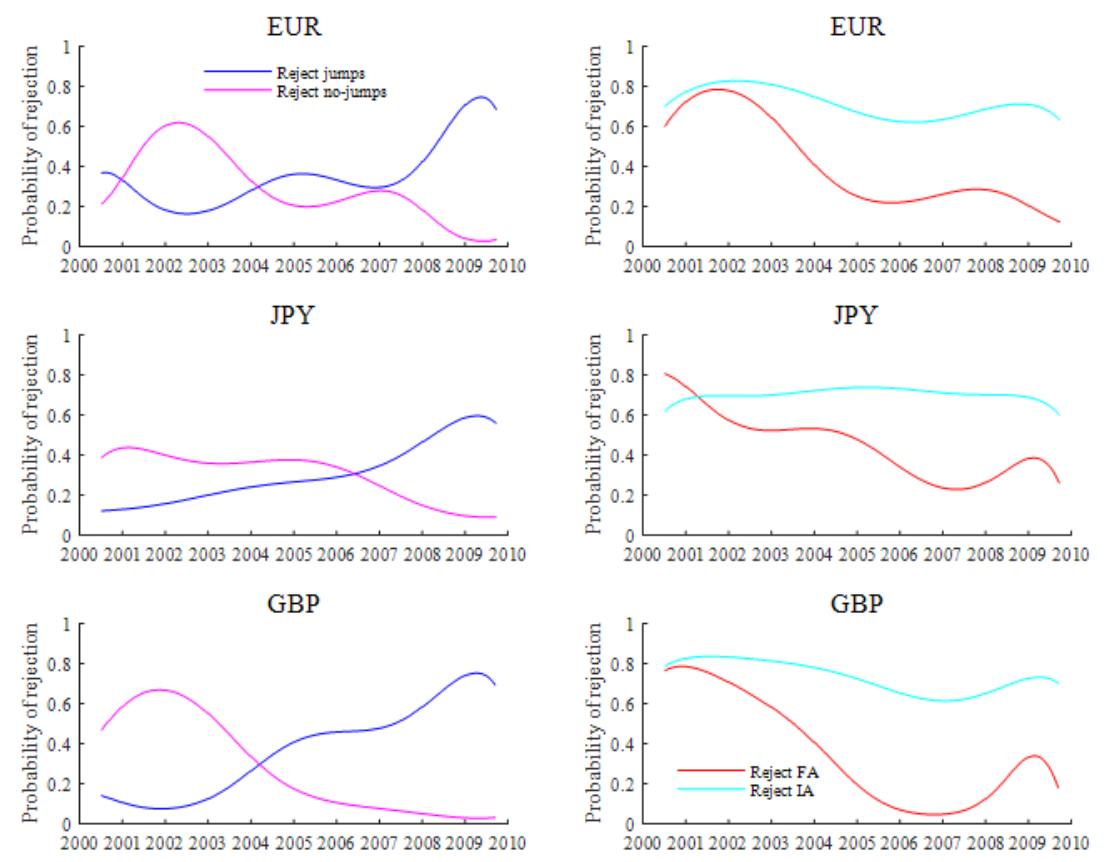

Figure 4: Time variation in rejection rates predicted by a probit model with a 6th order polynomial in time. The $Y$-axis denotes the probability of rejections over time. The sample covers January 1, 2000 to March 12, 2010 for EUR/USD, $U S D / J P Y$, and $G B P / U S D$. 